\title{
GRADIENT ESTIMATES VIA NON STANDARD POTENTIALS AND CONTINUITY
}

\author{
Verena Bögelein and Jens Habermann \\ Università di Parma, Dipartimento di Matematica \\ Viale Usberti 53/a, Campus, 43100 Parma, Italy; boegelein@mi.uni-erlangen.de \\ Università di Parma, Dipartimento di Matematica \\ Viale Usberti 53/a, Campus, 43100 Parma, Italy; habermann@mi.uni-erlangen.de
}

\begin{abstract}
We consider elliptic problems with non standard growth conditions whose most prominent model example is the $p(x)$-Laplacean equation

$$
-\operatorname{div}\left(|D u|^{p(x)-2} D u\right)=\mu,
$$

with a measure data right-hand side $\mu$. We prove pointwise gradient estimates in terms of a non standard version of the non-linear Wolff potential of the right-hand side measure, and moreover a characterization for $C^{1}$-regularity of the solution, also in terms of the Wolff potential. The $C^{1}$ regularity criterion is also related to the density of $\mu$ and the decay rate of its $L^{n}$-norm on small balls. Moreover, from the pointwise gradient estimates the Calderón and Zygmund theory and several types of local estimates follow as a consequence.
\end{abstract}

\section{Introduction and results}

In this paper we consider measure data problems under non standard growth conditions. Thereby, the most prominent model problem we have in mind is the $p(x)$-Laplacean equation

$$
-\operatorname{div}\left(|D u|^{p(x)-2} D u\right)=\mu,
$$

with a measure data right-hand side $\mu$. In this context we prove on the one hand pointwise gradient estimates in terms of the non-linear Wolff potential of the righthand side measure, and on the other hand a sufficient criterion for the $C^{1}$-regularity of the solution, also in terms of the Wolff potential. Since the pointwise gradient estimates provide a criterion for the solution to be Lipschitz continuous we are here in fact dealing with the borderline case between Lipschitz continuity and $C^{1}$-regularity. The search for possibly sharp bounds on the solution to PDEs and their regularity has an up to now long lasting tradition. Concerning the solution itself-not the gradient - these problems were solved for the case of standard growth in the by now classical works of Kilpeläinen and Malý [37, 38], and later extended with a different technique by Trudinger and Wang [51]. Similar results for elliptic operators with non standard growth conditions were achieved recently by Lukkari, Maeda and Marola [41], see also [6]. More precisely, the authors obtained a pointwise bound for the solutions to non-homogeneous partial differential equations of $p$-Laplacean,

doi:10.5186/aasfm.2010.3541

2000 Mathematics Subject Classification: Primary 35D10, 35J60, 35J70.

Key words: Pointwise gradient estimates, non standard Wolff potential, partial differential equations with non standard growth, measure data problems. 
respectively $p(x)$-Laplacean type in terms of a natural Wolff potential of the righthand side measure together with a continuity criterion. Surprisingly these results could recently be upgraded to the gradients of solutions. At first, a pointwise estimate for the gradient of solutions to equations with linear growth, i.e., the case $p=2$ was achieved by Mingione [45]. The full results dealing with the borderline case between Lipschitz continuity and $C^{1}$-regularity for solutions of general equations of $p$-Laplacean type with $p \geq 2$ were proved in fundamental papers by Duzaar and Mingione [16, 17, 18]. Note that these papers also contain similar results for $p$ Laplacean type systems with a diagonal structure.

Let us make some remarks concerning problems with $p(x)$ growth in general. Actually they are intensively studied in the literature for at least 15 years, attaining the interest of an increasing number of mathematicians for a variety of reasons. On one hand they represent the borderline case between standard $p$ growth and so-called $(p, q)$ growth conditions introduced in [42], therefore involving delicate perturbation arguments to treat the variable growth situation - of course assuming a certain regularity of the exponent function $p(\cdot)$. On the other hand a number of applications in mathematical physics, such as for example the modeling of non newtonian fluids - the most prominent example was given by Rajagopal and Růžička for electrorheological fluids in [47] — or image processing models quite recently established in [12], involve energies or systems of PDEs with non standard growth conditions. Typical structure conditions imposed on equations, systems or functionals with $p(x)$ growth in general allow to prove the existence of a (unique) solution or minimizer in the generalized Sobolev space $W^{1, p(\cdot)}(\Omega)$, defined by

$$
W^{1, p(\cdot)}(\Omega):=\left\{f: \Omega \rightarrow \mathbf{R}: \int_{\Omega}|f|^{p(x)} d x<+\infty \text { and } \int_{\Omega}|D f|^{p(x)} d x<+\infty\right\}
$$

Properties of those spaces, dependent on suitable more or less strong regularity assumptions on the exponent function $p(\cdot)$ have been intensively studied in the literature. The basic ones - such as reflexivity, separability, the availability of suitable versions of Sobolev's imbedding etc.- have been established in [19, 20, 34, 39]. The field of studying properties of those generalized spaces and their consequences is highly active and productive, just to mention recent contributions in [15, 30, 31, 32] and also the references therein.

Regularity theory for problems with $p(x)$ growth structure started with the essential paper [52] of Zhikov, who showed higher integrability, i.e., the existence of a quantity $\delta>0$ such that the solution belongs to the space $W_{\text {loc }}^{1, p(\cdot)(1+\delta)}(\Omega)$, provided that the exponent function $p(\cdot)$ fulfills a weak logarithmic continuity condition, in this paper expressed via (2.4). This turns out to be a starting point for any 'freezing procedure' which in turn allows to prove higher regularity via suitable comparison arguments. In $[1,2,13]$, this result was used to prove - under optimal conditions on the regularity of $p(x)$ - (partial) Hölder continuity results for minimizers and also their gradient. Note that in [24], Hölder continuity for solutions of equations was shown by a generalization of DeGiorgi's methods, under optimal assumptions on the regularity of $p(\cdot)$. We remark at this point that in order to guarantee that the solution $u$ of an equation of $p(x)$ growth type is Hölder continuous, i.e., to show $C^{0, \alpha_{-}}$ regularity, it is necessary to impose logarithmic Hölder continuity of the exponent 
function $p(\cdot)$ - expressed in our notation in (2.3) resp. (2.4), whereas to prove $C^{1, \alpha_{-}}$ regularity, i.e., Hölder continuity of the gradient of solutions, one needs to impose Hölder continuity of the exponent function $p(\cdot)$ itself. The initial essential papers on Hölder continuity were followed by a series of studies by a variety of authors, involving refinements covering also more general systems and functionals, inhomogeneities and boundary regularity, see for example [21, 23, 26, 28]. On the other hand, in [11] it was shown that a higher integrability result similar to the one of Zhikov also holds below the natural integrability exponent, in the sense that there exists $\varepsilon>0$ such that every so called very weak solution $u \in W^{1, p(\cdot)(1-\varepsilon)}(\Omega)$ already belongs to the natural Sobolev space $W_{\text {loc }}^{1, p(\cdot)}(\Omega)$ and then in turn is higher integrable by the result of Zhikov. A second kind of regularity treatments has been started by Acerbi and Mingione in [4], where they study quantified higher integrability statements for equations of $p(x)$ growth and the $p(x)$ Laplacean system. To prove results of that type, a delicate combination of Calderón-Zygmund type arguments with suitable freezing and comparison principles is needed. Also the techniques introduced there turn out to be quite flexible to treat also systems of more general structure (see [27]). Taking as a special case right hand sides $\equiv 0$, the results in [4] recover the $C^{0, \alpha}$-regularity, initially proved in [24] and [1]. To have an extensive outline about the state of the art concerning $p(x)$ growth problems, we refer the reader to the recent overwiew in [29].

In the present paper, we are actually dealing with a larger class of operators than the $p(x)$-Laplace operator, which includes this easiest model example. More precisely, we consider solutions to partial differential equations with non standard growth and measure data right-hand sides of the form

$$
-\operatorname{div} a(x, D u)=\mu .
$$

Here, $\mu$ denotes a Radon measure defined on a bounded domain $\Omega \subset \mathbf{R}^{n}, n \geq 2$, with finite total mass. The continuous vector field $a: \Omega \times \mathbf{R}^{n} \rightarrow \mathbf{R}^{n}$ is assumed to be $C^{1}$-regular in the gradient variable $z$, with $a_{z}(\cdot)$ being Carathéodory regular and satisfying the following non standard growth and ellipticity assumptions:

$$
\left\{\begin{array}{l}
|a(x, z)|+\left|a_{z}(x, z)\right|\left(|z|^{2}+s^{2}\right)^{\frac{1}{2}} \leq L\left(|z|^{2}+s^{2}\right)^{\frac{p(x)-1}{2}} \\
\nu\left(|z|^{2}+s^{2}\right)^{\frac{p(x)-2}{2}}|\lambda|^{2} \leq\left\langle a_{z}(x, z) \lambda, \lambda\right\rangle
\end{array}\right.
$$

whenever $x \in \Omega$ and $z, \lambda \in \mathbf{R}^{n}$, where $0<\nu \leq L$ and $s \in[0,1]$ are fixed. Additionally, we shall impose the following continuity assumption on $a(\cdot)$ with respect to $x$ : There exists $L_{1} \geq 1$ such that

$$
\begin{aligned}
& \left|a(x, z)-a\left(x_{0}, z\right)\right| \\
& \leq L_{1} \omega\left(\left|x-x_{0}\right|\right)\left[\left(|z|^{2}+s^{2}\right)^{\frac{p(x)-1}{2}}+\left(|z|^{2}+s^{2}\right)^{\frac{p\left(x_{0}\right)-1}{2}}\right]\left[1+\left|\log \left(|z|^{2}+s^{2}\right)\right|\right]
\end{aligned}
$$

holds for all $x, x_{0} \in \Omega$ and $z \in \mathbf{R}^{n}$. Moreover, the exponent function $p: \Omega \rightarrow[2,+\infty)$ is assumed to be continuous with modulus of continuity $\omega:[0, \infty) \rightarrow[0,1]$, i.e., there holds

$$
2 \leq p(x) \leq \gamma_{2} \quad \text { and } \quad|p(x)-p(y)| \leq \omega(|x-y|),
$$

for all $x, y \in \Omega$. Let us note at this stage that we can always find such an upper bound $\gamma_{2}$ of $p(\cdot)$ since $\Omega$ is bounded and $p(\cdot)$ is continuous with modulus of continuity 
$\omega$. Moreover, since the results in this paper are of local nature, one may always replace $\gamma_{2}$ in (1.5) and also in (1.6) below by the maximal exponent in the considered neighborhood of some point $x_{0} \in \Omega$. Finally, for the modulus of continuity $\omega$ we assume that it is a non-decreasing concave function such that $\omega(0)=0=\lim _{\varrho \downarrow 0} \omega(\varrho)$, satisfying a certain asymptotic smallness condition when $\varrho \downarrow 0$ of the form: there exists $\varrho>0$ such that

$$
\int_{0}^{\varrho}\left[\omega(r) \log \frac{1}{r}\right]^{2 / \gamma_{2}} \frac{d r}{r}=: d(\varrho)<\infty
$$

holds. This actually is the condition under which we shall prove continuity of the gradient of the solution. The precise condition under which we shall achieve our local gradient potential estimate is slightly weaker and takes the form (1.9). Note that condition (1.9) on $\omega$ indeed depends on the particular point $x_{0} \in \Omega$ considered, or more precisely on $p\left(x_{0}\right)$. Note also that (1.6) actually is implied by (1.9), and therefore all results continue to hold under the assumption (1.6).

The estimates proved in this paper involve the following non-linear Wolff potential for variable exponent functions

$$
\mathbf{W}_{\beta(\cdot), p(\cdot)}^{\mu}(x, R):=\int_{0}^{R}\left(\frac{|\mu|\left(B_{\varrho}(x)\right)}{\varrho^{n-\beta(x) p(x)}}\right)^{\frac{1}{p(x)-1}} \frac{d \varrho}{\varrho}, \quad \beta(x) \in(0, n / p(x)],
$$

which is defined pointwise just as the usual constant exponent Wolff potential. Within the whole paper we shall assume that the total 1-energy of the solution $u$ is bounded, i.e.,

$$
\int_{\Omega}|D u| d x=: M<+\infty .
$$

Note that we cannot use the $p(\cdot)$-energy instead - as it is usually done for non standard growth problems - for the following reason: Although we prove the gradient estimate in Theorem 1.1 as an a priori estimate and therefore assume that the solution is of class $C^{1}$ we shall later apply it to weak solutions and even to so called SOLAs in Theorem 1.4, and for SOLAs we only know that $D u \in L^{p(\cdot)-1}$. Therefore, all constants in the estimates should be independent of the $p(\cdot)$-energy of $u$.

1.1. Gradient estimates. The first main result of the paper is the following gradient potential estimate which is stated as an a priori estimate.

Theorem 1.1. Let $u \in C^{1}(\Omega)$ be a weak solution of (1.2) with $\mu \in L^{1}(\Omega)$ under the assumptions (1.3) and (1.4). Then, there exists a constant $c \equiv c\left(n, \nu, L, \gamma_{2}, M\right.$, $|\mu|(\Omega))$ such that the following is true: for any $x_{0} \in \Omega$ satisfying

$$
\int_{0}^{\varrho}\left[\omega(r) \log \frac{1}{r}\right]^{2 / p\left(x_{0}\right)} \frac{d r}{r}=: d\left(x_{0}, \varrho\right)<\infty \quad \text { for some } \varrho>0,
$$

there exists a positive radius $R_{0} \equiv R_{0}\left(n, \nu, L, L_{1}, \gamma_{2}, M,|\mu|(\Omega), \omega(\cdot), p\left(x_{0}\right)\right)$ such that the pointwise estimate

$$
\left|D u\left(x_{0}\right)\right| \leq c f_{B_{R}\left(x_{0}\right)}(|D u|+s) d x+c \mathbf{W}_{\frac{1}{p(\cdot)}, p(\cdot)}^{\mu}\left(x_{0}, 2 R\right)+c R
$$

holds whenever $B_{2 R}\left(x_{0}\right) \subseteq \Omega$ with $R \leq R_{0}$. Moreover, under the stronger assumption (1.6) instead of (1.9) the radius $R_{0}$ is independent of $p\left(x_{0}\right)$. 
Remark 1.2. Note that the dependence of the constant $c$ and the radius $R_{0}$ upon $|\mu|(\Omega)$ in Theorem 1.1 is not unavoidable. By a slightly more involved argument we could get rid of this dependence. Indeed, keeping the dependence $|\mu|(\Omega)$ in all estimates explicit it turns out that in Lemma 3.5 we would obtain a term $\left[|\mu|\left(B_{2 R}\right) / R^{n-1}\right]^{\frac{1}{p_{0}-1}+c \omega(4 R)}$ instead of $\left[|\mu|\left(B_{2 R}\right) / R^{n-1}\right]^{\frac{1}{p_{0}-1}}$ on the right-hand side, where $\omega$ is the modulus of continuity of the exponent function $p(\cdot)$. Then, in the proof of Theorem 1.1 we can modify (3.31) in the following way:

$$
\begin{aligned}
& f_{B_{R / H}}\left|D u-(D u)_{B_{R / H}}\right| d x \\
& =\left[f_{B_{R / H}}\left|D u-(D u)_{B_{R / H}}\right| d x\right]^{\frac{c \omega(4 R)}{1+c \omega(4 R)}}\left[f_{B_{R / H}}\left|D u-(D u)_{B_{R / H}}\right| d x\right]^{\frac{1}{1+c \omega(4 R)}} \\
& \leq c(L, M)\left[f_{B_{R / H}}\left|D u-(D u)_{B_{R / H}}\right| d x\right]^{\frac{1}{1+c \omega(4 R)}},
\end{aligned}
$$

by the localization argument (2.7) explained below. The remaining integral is then similarly estimated as in (3.31). This procedure leads to a final constant independent of $|\mu|(\Omega)$. However, for the sake of readability we did not carry this out and stated the dependence of the constants as in Theorem 1.1.

Although our gradient estimate in Theorem 1.1 is stated for $C^{1}$-solutions it can be carried over to general solutions of measure data problems with non standard growth via an approximation procedure see Chapter 4. In the particular case that $\mu \in$ $W^{-1, p^{\prime}(\cdot)}(\Omega)$, where $W^{-1, p^{\prime}(\cdot)}$ denotes the dual space of $W^{1, p(\cdot)}$ we know that-under suitable assumptions on the boundary data - there exists a unique weak solution $u \in W^{1, p(\cdot)}(\Omega)$ of Dirichlet problems associated to (1.2). In this case we have the following

Theorem 1.3. Let $u \in W^{1, p(\cdot)}(\Omega)$ be a weak solution of $(1.2)$ with $\mu \in W^{-1, p^{\prime}(\cdot)}(\Omega)$ under the assumptions (1.3) and (1.4). Then, the assertion of Theorem 1.1 holds, for almost every $x_{0} \in \Omega$. Moreover, under the stronger assumption (1.6) instead of (1.9) we have

$$
\mathbf{W}_{\frac{1}{p(\cdot)}, p(\cdot)}^{\mu}(\cdot, R) \in L^{\infty}\left(\Omega_{0}\right) \text { for some } R>0, \Omega_{0} \subseteq \Omega \Longrightarrow D u \in L_{\mathrm{loc}}^{\infty}\left(\Omega_{0}, \mathbf{R}^{n}\right),
$$

and there exists a constant $c \equiv c\left(n, \nu, L, L_{1}, \gamma_{2}, M,|\mu|(\Omega), \omega(\cdot)\right)$ such that

$$
\|D u\|_{L^{\infty}\left(B_{R / 2}\left(x_{0}\right)\right)} \leq c f_{B_{R}\left(x_{0}\right)}(|D u|+s) d x+c\left\|\mathbf{W}_{\frac{1}{p(\cdot)}, p(\cdot)}^{\mu}(\cdot, R)\right\|_{L^{\infty}\left(B_{R / 2}\left(x_{0}\right)\right)}+c R .
$$

In the more general case that the right-hand side $\mu$ is merely a Radon measure with finite total mass, we shall consider Dirichlet problems of the type

$$
\begin{cases}-\operatorname{div} a(x, D u)=\mu & \text { in } \Omega, \\ u=0 & \text { on } \partial \Omega .\end{cases}
$$

In this case we cannot work with the notion of a weak solution in the sense of Theorem 1.3 since they are in general not known to exist, even not for coefficients with constant exponent growth. Therefore, the notion of a solution has to be weakened and in the literature there have been introduced different approaches. We shall follow the one in $[9,10,14]$ of a so called SOLA (Solution Obtained by Limit of 
Approximations). These generalized solutions are very weak in the sense that they do not necessarily lie in the natural Sobolev space $W^{1, p(\cdot)}(\Omega)$ associated with the problem. On the other hand, they do belong to $W^{1, p(\cdot)-1}(\Omega)$ which gives a meaning to the weak formulation of (1.11). Moreover, in the case that $\mu \in L^{1}(\Omega)$ the SOLA is unique and when $\mu \in W^{-1, p^{\prime}(\cdot)}(\Omega)$ it coincides with the usual weak solution. For more details we refer to Section 4.1. Nevertheless, our gradient estimate (1.10) also extends to the most general case where $\mu$ is only a Radon measure and this is the statement of the following

Theorem 1.4. Let $u \in W^{1, p(\cdot)-1}(\Omega)$ be a SOLA of the Dirichlet problem (1.11) with $\mu$ being a Radon measure defined on $\Omega$ with finite total mass, under the assumptions (1.3) and (1.4). Then, the conclusions of Theorem 1.3 hold true.

In the case when $\mu \in L^{n}$ the pointwise gradient bound directly relies on the decay rate of $r \mapsto\|\mu\|_{L^{n}\left(B_{r}\left(x_{0}\right)\right)}$ when $r \downarrow 0$. This is expressed in the following

Theorem 1.5. Let $u \in W^{1, p(\cdot)}(\Omega)$ be a weak solution of (1.2) under the assumptions (1.3) and (1.4) and suppose that $\mu \in L^{n}(\Omega)$. Then, there exists a constant $c \equiv$ $c\left(n, \nu, L, \gamma_{2}, M,|\mu|(\Omega)\right)$ such that the following is true: for almost every $x_{0} \in \Omega$ satisfying (1.9) there exists a positive radius $R_{0} \equiv R_{0}\left(n, \nu, L, L_{1}, \gamma_{2}, M,|\mu|(\Omega), \omega(\cdot), p\left(x_{0}\right)\right)$ such that the pointwise estimate

$$
\left|D u\left(x_{0}\right)\right| \leq c f_{B_{R}\left(x_{0}\right)}(|D u|+s) d x+c \int_{0}^{2 R}\|\mu\|_{L^{n}\left(B_{r}\left(x_{0}\right)\right)}^{\frac{1}{p\left(x_{0}\right)-1}} \frac{d r}{r}+c R
$$

holds whenever $B_{2 R}\left(x_{0}\right) \subseteq \Omega$ with $R \leq R_{0}$.

The proof of the a priori estimate in Theorem 1.1 will be given in Chapter 3 , whereas the proofs of Theorems 1.3 and 1.4 can be found in Chapter 4 and the one of Theorem 1.5 in Section 5.1.

1.2. Zero order estimates. Zero order pointwise estimates for solutions via Wolff potentials have been widely investigated up to now. Starting from [37, 38] which are dealing with the constant exponent case, the variable exponent case has been considered in [41, Theorem 4.3], where the following estimate for superharmonic functions and non-negative measures has been shown:

$$
\left|u\left(x_{0}\right)\right| \leq c\left[f_{B_{R}\left(x_{0}\right)}|u|^{\gamma} d x\right]^{\frac{1}{\gamma}}+c \mathbf{W}_{1, p(\cdot)}^{\mu}\left(x_{0}, 2 R\right)+c R,
$$

for $R$ small enough and $\gamma$ large enough depending on $n$ and $p(\cdot)$. Our results upgrade such an estimate to the gradient of the solution, replacing $\mathbf{W}_{1, p(\cdot)}^{\mu}\left(x_{0}, 2 R\right)$ by $\mathbf{W}_{\frac{1}{p(\cdot)}, p(\cdot)}^{\mu}\left(x_{0}, 2 R\right)$ which is natural by the scaling properties of the Wolff potential. On the other hand, our method also produces a zero order estimate, similar to (1.12) even with $\gamma=1$-and updates it for general signed measures. For more details we refer to Section 5.3.

1.3. $C^{\mathbf{1}}$-regularity. For the pointwise gradient potential estimate in Theorem 1.1 we only needed the boundedness of the Wolff potential in this point. But the behavior of the Wolff potential also provides us with a sufficient criterion for the gradient of the solution to be continuous. More precisely, if the Wolff potential satisfies a certain uniform decay property, then we can show that the solution is of class $C^{1}$. 
Theorem 1.6. Let $u \in W^{1, p(\cdot)}(\Omega)$ be a weak solution of (1.2) under the assumptions (1.3), (1.4) and (1.6). Assumed that the functions

$$
x \mapsto \mathbf{W}_{\frac{1}{p(\cdot)}, p(\cdot)}^{\mu}(x, \varrho) \text { converge locally uniformly to zero in } \Omega \text { as } \varrho \downarrow 0,
$$

then $D u$ is continuous in $\Omega$.

Note that (1.13) satisfied on $\Omega$ implies by a variable exponent version of the Hedberg-Wolff Theorem - see Theorem 2.1 - that $\mu \in W^{-1, p(\cdot)}(\Omega)$ ensuring the existence of a $W^{1, p(\cdot)}$ solution and therefore the assumption $u \in W^{1, p(\cdot)}(\Omega)$ in the previous theorem is not too restrictive. However, by the approximation argument in Section 4.1 we could state Theorem 1.6 for a SOLA as well. An immediate consequence of Theorem 1.6 concerns measures with a certain density property.

Theorem 1.7. Let $u \in W^{1, p(\cdot)}(\Omega)$ be a weak solution of (1.2) under the assumptions (1.3), (1.4) and (1.6). Assumed that there exists a function $h:[0, \infty) \rightarrow[0, \infty)$ satisfying

$$
\int_{0}^{\varrho} h(r) \frac{d r}{r}<\infty \quad \text { for some } \varrho>0
$$

and

$$
\left|\mu\left(B_{r}\left(x_{0}\right)\right)\right| \leq r^{n-1} h(r)^{\frac{1}{p\left(x_{0}\right)-1}} \quad \text { for every ball } B_{r}\left(x_{0}\right) \subseteq \Omega \text { with } r \leq R,
$$

then $D u$ is continuous in $\Omega$.

Moreover, when $\mu \in L^{n}$, then the gradient continuity can be directly related to the decay properties of $\varrho \mapsto\|\mu\|_{L^{n}\left(B_{\varrho}\left(x_{0}\right)\right)}$, in a similar way as we did for the gradient potential estimate in Theorem 1.5.

Theorem 1.8. Let $u \in W^{1, p(\cdot)}(\Omega)$ be a weak solution of (1.2) under the assumptions (1.3), (1.4) and (1.6) and suppose that $\mu \in L^{n}(\Omega)$. Assumed that the functions

$$
x \mapsto \int_{0}^{\varrho}\|\mu\|_{L^{n}\left(B_{r}(x)\right)}^{\frac{1}{p(x)-1}} \frac{d r}{r} \text { converge locally uniformly to zero in } \Omega \text { as } \varrho \downarrow 0,
$$

then $D u$ is continuous in $\Omega$.

Acknowledgements. This research is supported by the ERC grant 207573 "Vectorial Problems".

\section{Preliminaries}

2.1. Monotonicity of the vectorfield $\boldsymbol{a}(\cdot)$. At this point we recall that assumption $(1.3)_{2}$ implies the following monotonicity property of the vector field $z \mapsto a(\cdot, z)$ : There exists a constant $c \equiv c\left(\gamma_{2}\right) \geq 1$ such that

$$
c^{-1} \nu\left(\left|z_{1}\right|^{2}+\left|z_{2}\right|^{2}+s^{2}\right)^{\frac{p(x)-2}{2}}\left|z_{2}-z_{1}\right|^{2} \leq\left\langle a\left(x, z_{2}\right)-a\left(x, z_{1}\right), z_{2}-z_{1}\right\rangle,
$$

whenever $x \in \Omega$ and $z_{1}, z_{2} \in \mathbf{R}^{n}$. In particular, since $p(x) \geq 2$, the previous inequality directly implies

$$
c^{-1} \nu\left|z_{2}-z_{1}\right|^{p(x)} \leq\left\langle a\left(x, z_{2}\right)-a\left(x, z_{1}\right), z_{2}-z_{1}\right\rangle .
$$


2.2. The exponent function $\boldsymbol{p}(\cdot)$. We note that assumptions (1.6), respectively (1.9) prescribe some kind of logarithmic Dini continuity of the exponent function $p(\cdot)$. Of course this condition implies the strong logarithmic continuity of $p(\cdot)$, i.e., for its modulus of continuity $\omega$ holds

$$
\limsup _{\varrho \downarrow 0} \omega(\varrho) \log \left(\frac{1}{\varrho}\right)=0,
$$

and therefore certainly also the weak logarithmic continuity

$$
\limsup _{\varrho \downarrow 0} \omega(\varrho) \log \left(\frac{1}{\varrho}\right)<+\infty,
$$

which is an essential condition in many regularity proofs for problems with non standard growth structure in the literature, especially for gaining higher integrability in the spirit of Lemma 3.2, which is a starting point for any freezing procedure. We note at this point that very recently, Zhikov and Pastukhova [53] proved certain logarithmic type higher integrability results under slightly weaker conditions on the modulus of continuity of $p(\cdot)$. By virtue of (2.3), respectively (2.4) - eventually enlarging the upper bound $L$ in the structure conditions $(1.3)_{1}$ of the vector field $a(\cdot)$-we may assume that there exists $R_{0}=R_{0}(L, \omega(\cdot))$ such that

$$
\omega(R) \log \left(\frac{1}{R}\right) \leq L,
$$

for all $R \in\left(0, R_{0}\right]$. For a fixed ball $B_{R}\left(x_{0}\right) \subset \Omega$ we define

$$
p_{1}:=\inf _{x \in B_{R}\left(x_{0}\right)} p(x) \text { and } p_{2}:=\sup _{x \in B_{R}\left(x_{0}\right)} p(x) .
$$

Then, assumption (1.5) directly gives

$$
p_{2}-p_{1} \leq \omega(2 R) \quad \text { and } \quad \frac{p_{2}}{p_{1}} \leq 1+\omega(2 R) .
$$

Furthermore, an elementary computation shows that (2.5) and (2.6) imply

$$
R^{-\omega(R)} \leq c(L) \quad \text { and } \quad R^{-\left(p_{2}-p_{1}\right)} \leq c(L) .
$$

Finally, we infer an auxiliary estimate that we shall use several times in the course of the paper. For any $\alpha, \sigma>0, R \in\left(0, R_{0}\right]$ and $\tilde{\omega} \in[0, \omega(R)]$ we have

$$
A^{\sigma} \leq c(L, \alpha)\left(A+R^{\alpha}\right)^{\sigma+\tilde{\omega}} \text { for all } A \geq 0 .
$$

This inequality is a consequence of the following chain of inequalities using $(2.7)_{1}$ and the fact that $\tilde{\omega} \leq \omega(R)$ :

$$
\begin{aligned}
A^{\sigma} & \leq\left(A+R^{\alpha}\right)^{\sigma}=\left(A+R^{\alpha}\right)^{-\tilde{\omega}}\left(A+R^{\alpha}\right)^{\sigma+\tilde{\omega}} \\
& \leq R^{-\alpha \tilde{\omega}}\left(A+R^{\alpha}\right)^{\sigma+\tilde{\omega}} \leq c(L, \alpha)\left(A+R^{\alpha}\right)^{\sigma+\tilde{\omega}} .
\end{aligned}
$$

2.3. Non-linear potentials. The following variable exponent version of the Hedberg-Wolff theorem from [41, Theorem 5.3] provides a characterization of the space $W^{-1, p(\cdot)}$ in terms of the Wolff potential.

Theorem 2.1. Let $\Omega$ be a bounded set in $\mathbf{R}^{n}$ and $\mu$ a compactly supported Radon measure in $\Omega$. Then $\mu \in W_{0}^{-1, p(\cdot)}(\Omega)$ if and only if

$$
\int_{\Omega} \mathbf{W}_{1, p(\cdot)}^{\mu}(x, \varrho) d \mu(x)<\infty \quad \text { for some } \varrho>0 .
$$


2.4. A decay estimate below the natural growth exponent for a reference problem. The proof of the main theorem will be done via suitable comparisons to a "frozen" reference problem. For a sub-domain $A \subseteq \Omega$ and $x_{0} \in \Omega$ we consider a solution $w \in W^{1, p\left(x_{0}\right)}(A)$ to the homogeneous equation

$$
-\operatorname{div} a\left(x_{0}, D w\right)=0 \quad \text { in } A .
$$

Note that the considered vector field is frozen in the point $x_{0}$ and therefore satisfies growth and ellipticity conditions (1.3) with a fixed exponent $p\left(x_{0}\right)$ instead of $p(x)$. Therefore, [16, Theorem 3.1] applies to weak solutions of (2.9) and reads as follows:

Theorem 2.2. Let $w \in W^{1, p\left(x_{0}\right)}(A)$ be a weak solution to (2.9) under the assumptions (1.3) with $p(x) \equiv p\left(x_{0}\right) \equiv$ const. Then, there exist $\beta \in(0,1]$ and $c \geq 1$, both depending on $n, \nu, L, p\left(x_{0}\right)$, such that the estimate

$$
f_{B_{\varrho}}\left|D w-(D w)_{B_{\varrho}}\right| d x \leq c\left(\frac{\varrho}{R}\right)^{\beta} f_{B_{R}}\left|D w-(D w)_{B_{R}}\right| d x
$$

holds whenever $B_{\varrho} \subseteq B_{R}$ are concentric balls contained in $A$.

Remark 2.3. Note that the constants $\beta$ and $c$ in Theorem 2.2 depend on $p\left(x_{0}\right)$. However, since the dependence upon $p\left(x_{0}\right)$ is continuous and recalling that $p\left(x_{0}\right) \in$ $\left[2, \gamma_{2}\right]$, by possibly enlarging the constants we can choose them in such a way that they depend on $\gamma_{2}$ rather than on $p\left(x_{0}\right)$. At this stage we remark that the constants in [16, Theorem 3.1] are stable when $p \downarrow 2$ since they are relying on estimates for a linearized elliptic equation as considered in [16, Lemma 3.2].

\section{Proof of the gradient estimate}

The proof of the gradient potential estimate from Theorem 1.1 is done via comparison to a suitable homogeneous frozen problem, which provides good reference estimates. This comparison will be performed within two steps. First we shall compare the original inhomogeneous problem to the associated homogeneous problem and subsequently this homogeneous problem shall be compared to a homogeneous frozen coefficient problem. Within this procedure we shall consider the following Dirichlet problems. By $u \in C^{1}(\Omega)$ we shall denote a solution of the original problem (1.2) on $\Omega$ with a vector field $a(\cdot)$ satisfing (1.3) and (1.4) and with bounded 1-energy, i.e., (1.8) as considered in Theorem 1.1. Now, for a fixed ball $B_{2 R} \equiv B_{2 R}\left(x_{0}\right) \subseteq \Omega$ with suitably small radius $2 R$ - to be specified later - we consider the solution $v \in W^{1, p(\cdot)}(\Omega)$ of the Dirichlet problem

$$
\begin{cases}-\operatorname{div} a(x, D v)=0 & \text { on } B_{2 R}, \\ v=u & \text { on } \partial B_{2 R}\end{cases}
$$

and the solution $w \in W^{1, p_{0}}(\Omega)$, where $p_{0} \equiv p\left(x_{0}\right)$ of the Dirichlet problem

$$
\begin{cases}-\operatorname{div} a\left(x_{0}, D w\right)=0 & \text { on } B_{R} \\ w=v & \text { on } \partial B_{R} .\end{cases}
$$

Existence and uniqueness of $u$ and $v$ are guaranteed by standard monotonicity arguments, which can be done also in the generalized Sobolev space $W^{1, p(\cdot)}(\Omega)$. As mentioned above the first step is the 
3.1. Comparing to a homogeneous problem. Here, we shall derive a comparison estimate between the solutions $u$ of the original inhomogeneous problem (1.2) and the solution and $v$ of the associated homogeneous problem (3.1).

Lemma 3.1. Under the assumptions $(1.3)_{2}$, (1.5) and (2.4), let $u \in W^{1, p(\cdot)}(\Omega)$ be as in Theorem 1.1 and $v \in u+W_{0}^{1, p(\cdot)}\left(B_{2 R}\right)$ as in (3.1), with $B_{2 R} \equiv B_{2 R}\left(x_{0}\right) \subseteq \Omega$, $0<R \leq R_{0}$, where $R_{0} \equiv R_{0}(L, \omega(\cdot))$. Then, setting $p_{0} \equiv p\left(x_{0}\right)$ there exists a constant $c \equiv c\left(n, \nu, L, \gamma_{2},|\mu|(\Omega)\right)$ such that the following inequalities hold true:

$$
f_{B_{2 R}}|D u-D v| d x \leq c\left[\frac{|\mu|\left(B_{2 R}\right)}{R^{n-1}}\right]^{\frac{1}{p_{0}-1}}+c R,
$$

and

$$
f_{B_{2 R}}|u-v| d x \leq c\left[\frac{|\mu|\left(B_{2 R}\right)}{R^{n-p_{0}}}\right]^{\frac{1}{p_{0}-1}}+c R^{2} .
$$

Proof. The proof is divided into three steps. We first assume without loss of generality that $x_{0}=0$ and $2 R=1$, i.e., $B_{2 R}=B_{1}$ and also $|\mu|\left(B_{1}\right)=1$. The scaling technique allowing us to reduce to such a situation will be explained in the second step. Since this procedure shall lead us to a wrong exponent, i.e., $p_{2}=\sup _{x \in B_{2 R}} p(x)$ instead of $p_{0}$, we adjust the exponent in the final step.

Step 1: Dimensionless estimate. Here, we show that in the case $B_{2 R}=B_{1}$ and $|\mu|\left(B_{1}\right)=1$ we have

$$
\int_{B_{1}}|D u-D v| d x \leq c\left(n, \nu, \gamma_{2}\right) .
$$

For $k \in \mathbf{N}$ we define the following truncation operators

$$
T_{k}(t):=\max \{-k, \min \{t, k\}\} \quad \text { and } \quad \Phi_{k}(t):=T_{1}\left(t-T_{k}(t)\right),
$$

for $t \in \mathbf{R}$. We subtract the weak formulations of (1.2) and (3.1) and test the resulting equation

$$
\int_{B_{1}}\langle a(x, D u)-a(x, D v), D \varphi\rangle d x=\int_{B_{1}} \varphi d \mu
$$

with $\varphi:=T_{k}(u-v)$. Setting $D_{k}=\left\{x \in B_{1}:|u(x)-v(x)| \leq k\right\}$ we observe that $D \varphi=D u-D v$ on $D_{k}$, whereas $D \varphi=0$ on $B_{1} \backslash D_{k}$. Therefore, using (2.2) and the fact that $|\varphi| \leq k$ we infer that

$$
\int_{D_{k}}|D u-D v|^{p(x)} d x \leq c\left(\nu, \gamma_{2}\right) k .
$$

Since $k \geq 1$, this certainly implies

$$
\int_{D_{k}}|D u-D v| d x \leq \int_{D_{k}}(|D u-D v|+1)^{p(x)} d x \leq c\left(n, \nu, \gamma_{2}\right) k .
$$

Similarly, testing (3.6) with $\varphi=\Phi_{k}(u-v)$ yields

$$
\int_{C_{k}}|D u-D v|^{p(x)} d x \leq c\left(\nu, \gamma_{2}\right)
$$


where we have denoted $C_{k}:=\left\{x \in B_{1}: k<|u(x)-v(x)| \leq k+1\right\}$. This time we have used that $D \varphi=D u-D v$ on $C_{k}$, and $D \varphi=0$ on $B_{1} \backslash C_{k}$ and that $|\varphi| \leq 1$. Together with Hölder's inequality this implies

$$
\begin{aligned}
\int_{C_{k}}|D u-D v| d x & \leq\left|C_{k}\right|^{\frac{1}{2}}\left[\int_{C_{k}}|D u-D v|^{2} d x\right]^{\frac{1}{2}} \\
& \leq\left|C_{k}\right|^{\frac{1}{2}}\left[\int_{C_{k}}(|D u-D v|+1)^{p(x)} d x\right]^{\frac{1}{2}} \leq c\left(n, \nu, \gamma_{2}\right)\left|C_{k}\right|^{\frac{1}{2}} .
\end{aligned}
$$

From the very definition of $C_{k}$ we find

$$
\left|C_{k}\right| \leq k^{-\frac{n}{n-1}} \int_{C_{k}}|u-v|^{\frac{n}{n-1}} d x
$$

and hence

$$
\int_{C_{k}}|D u-D v| d x \leq c\left(n, \nu, \gamma_{2}\right) k^{-\frac{n}{2(n-1)}}\left[\int_{C_{k}}|u-v|^{\frac{n}{n-1}} d x\right]^{\frac{1}{2}} .
$$

Using (3.7) and (3.8) with $k_{0}$ being a fixed positive integer, and also Hölder's inequality for sequences and Sobolev's inequality we obtain

$$
\begin{aligned}
\int_{B_{1}}|D u-D v| d x & =\int_{D_{k_{0}}}|D u-D v| d x+\sum_{k=k_{0}}^{\infty} \int_{C_{k}}|D u-D v| d x \\
& \leq c k_{0}+c \sum_{k=k_{0}}^{\infty} k^{-\frac{n}{2(n-1)}}\left[\int_{C_{k}}|u-v|^{\frac{n}{n-1}} d x\right]^{\frac{1}{2}} \\
& \leq c k_{0}+c\left[\sum_{k=k_{0}}^{\infty} k^{-\frac{n}{n-1}}\right]^{\frac{1}{2}}\left[\int_{B_{1}}|u-v|^{\frac{n}{n-1}} d x\right]^{\frac{1}{2}} \\
& \leq c k_{0}+c H\left(k_{0}\right)\left[\int_{B_{1}}|D u-D v| d x\right]^{\frac{n}{2(n-1)}},
\end{aligned}
$$

where $c\left(n, \nu, \gamma_{2}\right)$ and in the last line we have set

$$
H\left(k_{0}\right):=\left[\sum_{k=k_{0}}^{\infty} k^{-\frac{n}{n-1}}\right]^{\frac{1}{2}}
$$

Note that $\frac{n}{n-1}>1$ and therefore $H\left(k_{0}\right)$ is finite and satisfies $H\left(k_{0}\right) \rightarrow 0$ as $k_{0} \rightarrow \infty$. Now, if $n>2$, we take $k_{0}=1$ in (3.9) and apply Young's inequality to absorb the integral of the right-hand side into the left to end up with (3.5). On the other hand, if $n=2$, we choose $k_{0}=k_{0}\left(n, \nu, \gamma_{2}\right)$ in (3.9) large enough such that $c H\left(k_{0}\right) \leq 1 / 2$ and again absorb the integral of the right-hand side into the left to deduce (3.5) also in this case.

Step 2: Scaling procedures. Here, we set

$$
p_{2}:=\sup _{x \in B_{2 R}} p(x)
$$


and show that there exists a constant $c \equiv c\left(n, \nu, \gamma_{2},|\mu|(\Omega)\right)$ such that

$$
f_{B_{2 R}}|D u-D v| d x \leq c\left[\frac{|\mu|\left(B_{2 R}\right)}{R^{n-1}}\right]^{\frac{1}{p_{2}-1}} .
$$

The assertion will follow from Step 1 where we assumed $B_{2 R}=B_{1}$ and $|\mu|\left(B_{1}\right)=1$ by two subsequent scaling procedures. We first reduce (3.10) to the case $B_{2 R}=B_{1}$ by a scaling argument. For $y \in B_{1}$ we let

$$
\begin{cases}\tilde{u}(y):=(2 R)^{-1} u\left(x_{0}+2 R y\right), & \tilde{v}(y):=(2 R)^{-1} v\left(x_{0}+2 R y\right), \\ \tilde{a}(y, z):=a\left(x_{0}+2 R y, z\right), & \tilde{\mu}(y):=2 R \mu\left(x_{0}+2 R y\right) .\end{cases}
$$

Then, $-\operatorname{div} \tilde{a}(y, D \tilde{u})=\tilde{\mu}$ and $-\operatorname{div} \tilde{a}(y, D \tilde{v})=0$, such that we can use estimate (3.10) for $\tilde{u}$ and $\tilde{v}$ on $B_{1}$ and then scale back to $B_{2 R}$.

In order to further reduce to the case $|\mu|\left(B_{1}\right)=1$ we adopt the following scaling. We define $A:=\left[|\tilde{\mu}|\left(B_{1}\right)\right]^{\frac{1}{p_{2}-1}}$ and note that we may assume $A>0$, since otherwise $\tilde{u} \equiv \tilde{v}$ and hence $u \equiv v$ and (3.10) holds trivially. Next, we set

$$
\begin{cases}\bar{u}(y):=A^{-1} \tilde{u}(y), & \bar{v}(y):=A^{-1} \tilde{v}(y), \\ \bar{a}(y, z):=A^{1-p_{2}} \tilde{a}(y, A z), & \bar{\mu}(y):=A^{1-p_{2}} \tilde{\mu}(y),\end{cases}
$$

such that $\bar{u}$ and $\bar{v}$ are solutions of $-\operatorname{div} \bar{a}(y, D \bar{u})=\bar{\mu}$ and $-\operatorname{div} \bar{a}(y, D \bar{v})=0$ on $B_{1}$. At this point we still have to ensure that we can apply (3.5) from Step 1 to $\bar{u}$ and $\bar{v}$. From the definition of $A$ we see that $|\bar{\mu}|\left(B_{1}\right)=1$. Therefore, it remains to ensure that $\bar{a}(\cdot)$ still satisfies $(1.3)_{2}$ (note that this is the only assumption on the vector field we used in Step 1). Due to the definition of $\bar{a}(\cdot)$ and the hypothesis $(1.3)_{2}$ on $a(\cdot)$ we have

$$
\begin{aligned}
\langle\bar{a}(x, z) \lambda, \lambda\rangle & =A^{1-p_{2}}\langle\tilde{a}(x, A z) \lambda, \lambda\rangle \geq \nu A^{1-p_{2}}\left(|A z|^{2}+s^{2}\right)^{\frac{p(x)-2}{2}}|\lambda|^{2} \\
& =\nu A^{p(x)-p_{2}}\left(|z|^{2}+(s / A)^{2}\right)^{\frac{p(x)-2}{2}}|\lambda|^{2} .
\end{aligned}
$$

From the definition of $\tilde{\mu}$ and (2.7) - assuming that $R \leq R_{0}$ with $R_{0} \equiv R_{0}(L, \omega(\cdot))$ from (2.5) - we have

$$
\begin{aligned}
A^{p(x)-p_{2}} & =|\tilde{\mu}|\left(B_{1}\right)^{-\frac{p_{2}-p(x)}{p_{2}-1}}=(2 R)^{\frac{(n-1)\left(p_{2}-p(x)\right)}{p_{2}-1}}|\mu|\left(B_{2 R}\right)^{-\frac{p_{2}-p(x)}{p_{2}-1}} \\
& \geq c(L)^{-\frac{n-1}{p_{2}-1}}(|\mu|(\Omega)+1)^{-\frac{p_{2}-p(x)}{p_{2}-1}} \geq c(n, L)^{-1}(|\mu|(\Omega)+1)^{-1},
\end{aligned}
$$

and therefore in turn

$$
\langle\bar{a}(x, z) \lambda, \lambda\rangle \geq \frac{\nu}{c(n, L)(|\mu|(\Omega)+1)}\left(|z|^{2}+(s / A)^{2}\right)^{\frac{p(x)-2}{2}}|\lambda|^{2},
$$

meaning that $\bar{a}(\cdot)$ satisfies $(1.3)_{2}$ with $(\nu, s)$ replaced by $(\nu /[c(|\mu|(\Omega)+1)], s / A)$. Therefore, we can apply (3.5) to $\bar{u}$ and $\bar{v}$ to have

$$
\int_{B_{1}}|D \bar{u}-D \bar{v}| d x \leq c\left(n, \nu, L, \gamma_{2},|\mu|(\Omega)\right) .
$$

Note that the constant in the preceding estimate indeed is independent of $A$ since the one in (3.5) does not depend on $s$. Recalling the definitions of $\bar{u}$ and $\bar{v}$ and $A$ we deduce estimate (3.10) for $u$ and $v$ from the previous one.

Step 3: Adjusting the exponent. In the final step we want to replace $p_{2}$ by $p_{0}$ in (3.10). Note that this causes difficulties only in the case $|\mu|\left(B_{2 R}\right) / R^{n-1} \leq 1$ since 
$\frac{1}{p_{2}-1} \leq \frac{1}{p_{0}-1}$. To this aim we use the auxiliary estimate (2.8) with the choices $A=$ $\left[|\mu|\left(B_{2 R}\right) / R^{n-1}\right]^{\frac{1}{p_{0}-1}}, \alpha=1, \sigma=\frac{p_{0}-1}{p_{2}-1}$ and $\tilde{\omega}=\frac{p_{2}-p_{0}}{p_{2}-1}$ (note that $\tilde{\omega} \leq p_{2}-p_{0} \leq \omega(4 R)$ by $(1.5)$ and $\sigma+\tilde{\omega}=1)$ to infer the following estimate for the right-hand side of (3.10):

$$
\left[\frac{|\mu|\left(B_{2 R}\right)}{R^{n-1}}\right]^{\frac{1}{p_{2}-1}} \leq c\left[\frac{|\mu|\left(B_{2 R}\right)}{R^{n-1}}\right]^{\frac{1}{p_{0}-1}}+c R,
$$

with a constant $c \equiv c(L)$. Using this estimate to further bound the right-hand side of (3.10) we deduce (3.3), i.e., the first assertion of the lemma. The second one, i.e., (3.4) follows from (3.3) via Poincaré's inequality since

$$
f_{B_{2 R}}|u-v| d x \leq c R f_{B_{2 R}}|D u-D v| d x \leq c\left[\frac{|\mu|\left(B_{2 R}\right)}{R^{n-p_{0}}}\right]^{\frac{1}{p_{0}-1}}+c R^{2} .
$$

This completes the proof of the lemma.

3.2. Higher integrability and energy bounds. In this section we shall establish suitable energy bounds for the solutions $v$ and $w$ of (3.1) and (3.2), which will be used later in the proof. Note that we do not have higher integrability for the solution $u$ of the original problem due to the missing regularity of the inhomogeneity $\mu$, being just an $L^{1}$-function. Nevertheless, the solution $v$ of the homogeneous problem shows at least local higher integrability properties, and so does the solution $w$ of the frozen homogeneous problem. This allows to establish energy bounds for $v$ and $w$. Let us start with a well known higher integrability result for the homogeneous problem, which goes back to Zhikov [52]. For the dependence of the constant we refer to Remark 3.3 below.

Lemma 3.2. Under the assumptions (1.3), (1.5) and (2.4), let $u \in W^{1, p(\cdot)}(\Omega)$ be as in Theorem 1.1. Then, there exists a radius $R_{0} \equiv R_{0}\left(n, L, \gamma_{2}, \omega(\cdot)\right)$ such that the following holds: let $v \in u+W_{0}^{1, p(\cdot)}\left(B_{2 R}\right)$ be the function defined in (3.1), with $B_{2 R} \equiv B_{2 R}\left(x_{0}\right) \subseteq \Omega, 0<R \leq R_{0}$. Then, there exists $\delta_{1} \equiv \delta_{1}\left(n, L / \nu, \gamma_{2}\right) \in(0,1]$ such that $|D v|^{p(\cdot)} \in L_{\mathrm{loc}}^{1+\delta_{1}}\left(B_{2 R}\right)$ and for any $\theta \in(0,1)$ and $\delta \in\left[0, \delta_{1}\right]$ the estimate

$$
\left[f_{B_{\theta \varrho}}(|D v|+s)^{p(x)(1+\delta)} d x\right]^{\frac{1}{1+\delta}} \leq c f_{B_{\varrho}}(|D v|+s+\varrho)^{p(x)} d x
$$

holds true whenever $B_{\varrho} \subseteq B_{3 R / 2}$. Note that $c \equiv c\left(n, L / \nu, \gamma_{2}, \theta, M,|\mu|(\Omega)\right)$, where $M$ was defined in (1.8), and $c \rightarrow \infty$ as $\theta \uparrow 1$.

Remark 3.3. Here, we have to make two remarks concerning Lemma 3.2. Firstly, although $s$ might be zero we can achieve on the right-hand side an additive constant " $\varrho$ " instead of " 1 " by using an estimate of the type of (2.8) whenever switching to a larger radius. Secondly, the constant in the usual higher integrability lemma (see for instance [52] and also the comments in [13]) initially depends on $\int_{B_{o}}|D v|^{p(x)} d x$ rather than on $M$ and $|\mu|(\Omega)$. To be precise, the constant depends on $\left(\int_{B_{\varrho}}|D v|^{p(x)} d x\right)^{\omega(2 \varrho)}$. In order to avoid this dependency we shall first explain how to estimate $\int_{B_{\varrho}}|D v|^{p(x)} d x$ in terms of $\int_{B_{2 R}}|D v| d x$. For this aim we start with a reverse Hölder inequality for $D v$ which can be achieved by testing the equation (3.1) with $v$ multiplied by a suitable cut-off function, as it is usually done in the proof of higher integrability (see [52]). 
More precisely, there exists a constant $c \equiv c\left(n, \nu, L, \gamma_{2}\right)$ such that

$$
\int_{B_{t_{1}}}(|D v|+s+R)^{p(x)} d x \leq \frac{c}{\left(t_{2}-t_{1}\right)^{\alpha}}\left[\int_{B_{t_{2}}}(|D v|+s+R)^{\frac{p_{1}}{\vartheta}} d x\right]^{\frac{\vartheta p_{2}}{p_{1}}}, \text { where } \vartheta=\sqrt{\frac{n+1}{n}},
$$

holds for concentric balls $B_{t_{1}} \subset B_{t_{2}}$ contained in $B_{2 R}$ and where we have set

$$
p_{1}:=\inf _{x \in B_{2 R}} p(x), \quad p_{2}:=\sup _{x \in B_{2 R}} p(x), \quad \alpha:=n\left(\frac{\vartheta p_{2}}{p_{1}}-1\right) .
$$

Applying Hölder's inequality to the right-hand side of the preceding inequality we obtain

$\int_{B_{t_{1}}}(|D v|+s+R)^{p(x)} d x \leq \frac{c}{\left(t_{2}-t_{1}\right)^{\alpha}}\left[\int_{B_{t_{2}}}(|D v|+s+R)^{p_{1}} d x\right]^{\beta}\left[\int_{B_{t_{2}}}(|D v|+s+R) d x\right]^{\gamma}$,

where we have set

$$
\beta:=\frac{p_{2}}{p_{1}} \cdot \frac{1-\frac{\vartheta}{p_{1}}}{1-\frac{1}{p_{1}}} \quad \text { and } \quad \gamma:=\frac{p_{2}}{p_{1}} \cdot \frac{\vartheta-1}{1-\frac{1}{p_{1}}} .
$$

Note that $\left(1-\frac{\vartheta}{p_{1}}\right) /\left(1-\frac{1}{p_{1}}\right) \leq\left(1-\frac{\vartheta}{\gamma_{2}}\right) /\left(1-\frac{1}{\gamma_{2}}\right)=c\left(n, \gamma_{2}\right)<1$ and therefore we can always choose $R_{0}$ in dependence on $n, \gamma_{2}$ and $\omega(\cdot)$ small enough in order to have $\beta \leq c\left(n, \gamma_{2}\right)<1$. Using $(2.8)$ and applying Young's inequality we obtain

$$
\begin{gathered}
\int_{B_{t_{1}}}(|D v|+s+R)^{p(x)} d x \leq \frac{c}{\left(t_{2}-t_{1}\right)^{\alpha}}\left[\int_{B_{t_{2}}}(|D v|+s+R)^{p(x)} d x\right]^{\beta}\left[\int_{B_{2 R}}(|D v|+s+R) d x\right]^{\gamma} \\
\leq \frac{1}{2} \int_{B_{t_{2}}}(|D v|+s+R)^{p(x)} d x+\frac{c}{\left(t_{2}-t_{1}\right)^{\frac{\alpha}{1-\beta}}}\left[\int_{B_{2 R}}(|D v|+s+R) d x\right]^{\frac{\gamma}{1-\beta}},
\end{gathered}
$$

where $c$ depends on $n, \nu, L$ and $\gamma_{2}$. Note that the preceding estimate particularly holds when $B_{\varrho} \subseteq B_{t_{1}} \subset B_{t_{2}} \subseteq B_{2 R}$ are concentric balls with $\varrho \leq t_{1}<t_{2} \leq 2 R$. At this stage we apply the iteration Lemma in [25, Lemma 6.1] to infer that

$$
\int_{B_{\varrho}}(|D v|+s+R)^{p(x)} d x \leq c(2 R-\varrho)^{-\frac{\alpha}{1-\beta}}\left[\int_{B_{2 R}}(|D v|+s+R) d x\right]^{\frac{\gamma}{1-\beta}},
$$

where $c \equiv c\left(n, \nu, L, \gamma_{2}\right)$. Next, we use the comparison estimate (3.3) from Lemma 3.1 and the fact that $\varrho \leq 3 R / 2$ to infer

$$
\begin{aligned}
& \int_{B_{\varrho}}(|D v|+s)^{p(x)} d x \\
& \leq c R^{-\frac{\alpha}{1-\beta}}\left[\int_{B_{2 R}}(|D u|+s+R) d x+R^{n}\left[\frac{|\mu|\left(B_{2 R}\right)}{R^{n-1}}\right]^{\frac{1}{p_{0}-1}}\right]^{\frac{\gamma}{1-\beta}} .
\end{aligned}
$$

Note that $R \leq 1$ and therefore also $R^{n-\frac{n-1}{p_{0}-1}} \leq 1, \gamma \leq c\left(n, \gamma_{2}\right)$ and since $\beta \leq$ $c\left(n, \gamma_{2}\right)<1$ we have $\frac{\gamma}{1-\beta} \leq c\left(n, \gamma_{2}\right)$. Therefore, we can bound the bracket $[\ldots]^{\frac{\gamma}{1-\beta}}$ on the right-hand side of the preceding inequality by a constant depending on $n, \gamma_{2}, M$ and $|\mu|(\Omega)$, such that it remains to consider the factor $\varrho^{-\frac{\alpha}{1-\beta}}$. First, we recall that $\beta \leq c\left(n, \gamma_{2}\right)<1$ and hence $\frac{\alpha}{1-\beta} \leq c\left(n, \gamma_{2}\right)$ by the definition of $\alpha$. Therefore, taking 
the preceding inequality to the power $\tilde{\omega}$ with some $0<\tilde{\omega} \leq \omega(4 R)$ we can use $(2.7)$ in order to conclude with

$$
\left[\int_{B_{\varrho}}(|D v|+s)^{p(x)} d x\right]^{\tilde{\omega}} \leq c\left(n, \nu, L, \gamma_{2}, M, \omega(\cdot),|\mu|(\Omega)\right),
$$

for any $\varrho \leq 3 R / 2$ such that $B_{\varrho} \subseteq B_{3 R / 2}$. By the argument at the beginning of the remark this allows us to choose the constant as indicated in the statement of the lemma.

In the following we suppose that the assumptions (1.3), (1.5) and (2.4) are in force and let $u \in C^{1}(\Omega)$ be be a weak solution of (1.2) as in Theorem 1.1. Moreover, let $B_{2 R} \equiv B_{2 R}\left(x_{0}\right) \subseteq \Omega$ and $v$ be the function defined in (3.1). In the sequel we will derive a suitable energy estimate for $v$. Thereby, initially $R$ is some arbitrary radius such that $0<R \leq 1$ and shall be successively be restricted to some smaller values $R_{0}$ within the proof of the energy estimate for $v$.

To derive the energy estimate for the solution $v$ to the Dirichlet problem (3.1) we first let $\delta_{1} \equiv \delta_{1}\left(n, L / \nu, \gamma_{2}\right) \in(0,1]$ denote the corresponding exponent from Lemma 3.2 and choose $R_{0} \in(0,1]$ such that $\omega\left(4 R_{0}\right) \leq \delta_{1} / 2$; then $R_{0} \equiv R_{0}\left(n, L / \nu, \gamma_{2}, \omega(\cdot)\right)$. From now on we consider $R \leq R_{0}$ and set

$$
p_{1}:=\inf _{x \in B_{2 R}} p(x) \quad \text { and } \quad p_{2}:=\sup _{x \in B_{2 R}} p(x) .
$$

By the choice of $R_{0}$ we find that

$$
p_{2}\left(1+\frac{\delta_{1}}{2}\right) \leq\left(p_{1}+\omega(4 R)\right)\left(1+\frac{\delta_{1}}{2}\right) \leq\left(p_{1}+\frac{\delta_{1}}{2}\right)\left(1+\frac{\delta_{1}}{2}\right) \leq p_{1}\left(1+\delta_{1}\right),
$$

and hence $p_{2}\left(1+\frac{\delta_{1}}{2}\right) \leq p(x)\left(1+\delta_{1}\right)$ for all $x \in B_{2 R}$. This implies under the assumptions of Lemma 3.2 that

$$
|D v| \in L^{p_{2}\left(1+\frac{\delta_{1}}{2}\right)}\left(B_{R}\right) .
$$

Moreover, from (2.8), applied with $\left(D v(x)+s, 2,1,\left(p(x)-p_{1}\right) / p_{1}\right)$ instead of $(A, \alpha, \sigma, \tilde{\omega})$, we infer for any $x \in B_{R}$ the pointwise estimate

$$
|D v(x)|+s \leq c(L)\left(|D v(x)|+s+R^{2}\right)^{\frac{p(x)}{p_{1}}} \leq 2 c(L)\left[(|D v(x)|+s)^{\frac{p(x)}{p_{1}}}+R^{2}\right] .
$$

Now, let $\sigma \in\left[p_{1}, p_{2}\left(1+\delta_{1} / 2\right)\right]$. Taking the preceding inequality to the power $\sigma$ and applying Lemma 3.2 with $\delta=\sigma / p_{1}-1$ which is allowed by (3.18) (and after eventually further reducing the value of $\left.R_{0}\right)$ we infer for any $\tilde{p} \in\left[p_{1}, p_{2}+\omega(4 R)\right]$ that

$$
\begin{aligned}
{\left[f_{B_{R}}(|D v|+s)^{\sigma} d x\right]^{\frac{\tilde{p}}{\sigma}} } & \leq c\left[f_{B_{R}}\left((|D v|+s)^{\frac{\sigma p(x)}{p_{1}}}+R^{2 \sigma}\right) d x\right]^{\frac{\tilde{p}}{\sigma}} \\
& \leq c\left[f_{B_{3 / 2 R}}(|D v|+s+R)^{p(x)} d x\right]^{\frac{\tilde{p}}{p_{1}}}+c R^{2 \tilde{p}} \\
& \leq c f_{B_{3 / 2 R}}(|D v|+s)^{p(x)} d x\left[f_{B_{3 / 2 R}}(|D v|+s)^{p(x)} d x\right]^{\frac{\tilde{p}-p_{1}}{p_{1}}}+c R^{2 \tilde{p}}
\end{aligned}
$$

with $c \equiv c\left(n, \nu, L, \gamma_{2}, M,|\mu|(\Omega)\right)$. At this stage we use (3.16) and (2.7) in order to bound the term $[\ldots]^{\frac{\hat{p}-p_{1}}{p_{1}}}$ by a constant depending on $n, \nu, L, \gamma_{2}, M$ and $|\mu|(\Omega)$. 
Therefore, we deduce that for any $\sigma \in\left[p_{1}, p_{2}\left(1+\delta_{1} / 2\right)\right]$ and $\tilde{p} \in\left[p_{1}, p_{2}+\omega(4 R)\right]$ there holds the following energy estimate for $v$ :

$$
\left[f_{B_{R}}(|D v|+s)^{\sigma} d x\right]^{\frac{\tilde{p}}{\sigma}} \leq c f_{B_{3 / 2 R}}(|D v|+s)^{p(x)} d x+c R^{2 \tilde{p}}
$$

where $c \equiv c\left(n, \nu, L, \gamma_{2}, M,|\mu|(\Omega)\right)$ and whenever $0<R \leq R_{0} \equiv R_{0}\left(n, L / \nu, \gamma_{2}, \omega(\cdot)\right)$.

3.3. Freezing the coefficients. At this stage we establish a comparison estimate between the solution $v$ of the homogeneous problem (3.1), and the solution $w$ of the frozen homogeneous one (3.2). More precisely, we will prove the following

Lemma 3.4. Under the assumptions of Theorem 1.1, let $B_{2 R} \equiv B_{2 R}\left(x_{0}\right) \subseteq \Omega$, $p_{0} \equiv p\left(x_{0}\right)$, and denote $v$ the solution of (3.1) on $B_{2 R}$ and by $w$ the solution of (3.2) on $B_{R}$. Then, there exists a constant $c \equiv c\left(n, \nu, L, \gamma_{2}, M,|\mu|(\Omega)\right)$ and a radius $R_{0} \equiv R_{0}\left(n, L / \nu, \gamma_{2}, \omega(\cdot)\right) \in(0,1]$ such that whenever $0<R \leq R_{0}$ the following estimate holds:

$$
f_{B_{R}}|D v-D w|^{p_{0}} d x \leq c L_{1}^{2} \omega^{2}(R)\left[\log ^{2}\left(\frac{1}{R}\right) f_{B_{3 / 2 R}}(|D v|+s)^{p(x)} d x+R^{p_{0}}\right] .
$$

Proof. By $\delta_{1} \equiv \delta_{1}\left(n, L / \nu, \gamma_{2}\right) \in(0,1]$ we denote the constant from Lemma 3.2. In a first step, we let $R_{0} \equiv R_{0}\left(n, L / \nu, \gamma_{2}, \omega(\cdot)\right)$ be the radius chosen in such a way that $\omega\left(4 R_{0}\right) \leq \delta_{1} / 4$. This ensures that (3.20) is applicable whenever $R \leq R_{0}$; note that we shall reduce the value of $R_{0}$ in the remainder of the proof. Finally, by $p_{1}$ and $p_{2}$ we go on denoting the minimal and maximal value of $p(\cdot)$ on $B_{2 R}$ as defined in (3.17). Now, using the monotonicity $(2.2)$ of the frozen vector field $a\left(x_{0}, \cdot\right)$, the weak formulation of equations (3.1) and (3.2), the continuity assumption (1.4) of the maping $x \mapsto a(x, \cdot)$, Young's inequality and noting that $\left|\log \left(|D u|^{2}+s^{2}\right)\right| \leq \log 2+2|\log (|D u|+s)|$ we deduce

$$
\begin{aligned}
& \frac{\nu}{c\left(\gamma_{2}\right)} f_{B_{R}}\left(|D v|^{2}+|D w|^{2}+s^{2}\right)^{\frac{p_{0}-2}{2}}|D v-D w|^{2} d x \\
\leq & f_{B_{R}}\left\langle a\left(x_{0}, D w\right)-a\left(x_{0}, D v\right), D w-D v\right\rangle d x \\
= & f_{B_{R}}\left\langle a(x, D v)-a\left(x_{0}, D v\right), D w-D v\right\rangle d x \\
\leq & L_{1} \omega(R) f_{B_{R}}\left[\left(|D v|^{2}+s^{2}\right)^{\frac{p(x)-1}{2}}+\left(|D v|^{2}+s^{2}\right)^{\frac{p_{0}-1}{2}}\right] \\
& \cdot\left[1+\left|\log \left(|D v|^{2}+s^{2}\right)\right|\right]|D w-D v| d x \\
\leq & \frac{\nu}{2 c\left(\gamma_{2}\right)} f_{B_{R}}\left(|D v|^{2}+s^{2}\right)^{\frac{p_{0}-2}{2}}|D v-D w|^{2} d x+\frac{c\left(\gamma_{2}\right) L_{1}^{2} \omega(R)^{2}}{\nu} \\
& \cdot f_{B_{R}}\left[\left(|D v|^{2}+s^{2}\right)^{\frac{2 p(x)-p_{0}}{4}}+\left(|D v|^{2}+s^{2}\right)^{\frac{p_{0}}{4}}\right]^{2}[1+|\log (|D v|+s)|]^{2} d x .
\end{aligned}
$$

Since $p_{0} \geq 2$ we can absorb the first term of the right-hand side into the left, and moreover, we can bound the integral on the left-hand side from below by $f_{B_{R}} \mid D v-$ 
$\left.D w\right|^{p_{0}}$. Proceeding in this way and rearranging terms leads us to

$$
\begin{aligned}
& f_{B_{R}}|D v-D w|^{p_{0}} d x \\
& \leq c L_{1}^{2} \omega(R)^{2}\left[f_{B_{R}}(|D v|+s)^{2 p(x)-p_{0}}[1+|\log (|D v|+s)|]^{2} d x\right. \\
& \left.\quad+f_{B_{R}}(|D v|+s)^{p_{0}}[1+|\log (|D v|+s)|]^{2} d x\right],
\end{aligned}
$$

where $c \equiv c\left(\nu, \gamma_{2}\right)$. In order to estimate the integrals on the right-hand side of (3.21) we shall first deduce a pointwise bound of the expression

$$
\mathscr{V}_{\tilde{p}}(x):=(|D v(x)|+s)^{\tilde{p}}[1+|\log (|D v(x)|+s)|]^{2} \text { for } \tilde{p} \in\left[p_{1}-\omega(4 R), p_{2}+\omega(4 R)\right] .
$$

Note that $\tilde{p}=2 p(x)-p_{0}$ and $\tilde{p}=p_{0}$ both lie in the admissible range and then $\mathscr{V}_{2 p(x)-p_{0}}$ is just the integrand in the first integral on the right-hand side of (3.21), while $\mathscr{V}_{p_{0}}$ is the integrand in the second one. For the pointwise estimate of $\mathscr{V}_{\tilde{p}}$ we now distinguish three cases: In the case $|D v(x)|+s \geq 1$, we have

$$
\mathscr{V}_{\tilde{p}}(x) \leq 4(|D v(x)|+s)^{p_{2}+\omega(4 R)} \log ^{2}\left(e+(|D v|+s)^{p_{2}+\omega(4 R)}\right) .
$$

When $|D v(x)|+s \in\left(R^{4}, 1\right)$ we have $|\log (|D v(x)|+s)| \leq \log R^{-2}=4 \log \left(\frac{1}{R}\right)$. Moreover, by reducing $R_{0}$ such that $R_{0} \leq 1$ /e we find that $\log 1 / R \geq 1$ such that $1+|\log (|D v(x)|+s)| \leq 5 \log \left(\frac{1}{R}\right)$. Subsequently using that $(|D v(x)|+s)^{\tilde{p}-p(x)} \leq 1$ when $\tilde{p} \geq p(x)$, while $(|D v(x)|+s)^{\tilde{p}-p(x)} \leq R^{-4(p(x)-\tilde{p})} \leq R^{-4\left(p(x)+\omega(4 R)-p_{1}\right)} \leq c(L)$ when $\tilde{p}<p(x)$ by $(2.7)$, we get

$$
\mathscr{V}_{\tilde{p}}(x) \leq 25 \log ^{2}\left(\frac{1}{R}\right)(|D v(x)|+s)^{\tilde{p}} \leq c(L) \log ^{2}\left(\frac{1}{R}\right)(|D v(x)|+s)^{p(x)} .
$$

Finally, in the case $|D v(x)|+s \leq R^{4}$ we have $1+|\log (|D v(x)|+s)| \leq 2 \mid \log (|D v(x)|+$ $s) \mid$ since $R \leq R_{0} \leq 1 / e$. In turn using the fact that the mapping $[0,1] \ni \xi \mapsto \xi^{\frac{1}{2}} \log ^{2} \xi$ attains its absolute maximum value $16 \mathrm{e}^{-2}$ in the point $\xi=\mathrm{e}^{-4}$, we conclude that $(|D v(x)|+s) \log ^{2}(|D v(x)|+s) \leq 16 e^{-2}$ and therefore

$$
\begin{aligned}
\mathscr{V}_{\tilde{p}}(x) & \leq 4(|D v(x)|+s)^{\tilde{p}} \log ^{2}(|D v(x)|+s) \leq 64 e^{-2}(|D v(x)|+s)^{\tilde{p}-\frac{1}{2}} \\
& \leq 16 R^{4\left(\tilde{p}-\frac{1}{2}\right)} \leq 16 R^{p_{0}},
\end{aligned}
$$

where we have also taken into account that $R \leq 1$ and $\tilde{p}-\frac{1}{2} \geq p_{1}-\omega(4 R)-\frac{1}{2} \geq$ $p_{1}-1 \geq p_{1} / 2 \geq p_{2} / 4 \geq p_{0} / 4$ by (2.6). At this point we can proceed the estimate of (3.21). More precisely, decomposing in the integrals on the right-hand side the domain of integration $B_{R}$ into the three disjoint sets where $|D v(x)|+s$ takes a value in $[1, \infty)$, respectively in $\left(R^{4}, 1\right)$ or in $\left[0, R^{4}\right]$ and using the according estimates from above with $\tilde{p}=2 p(x)-p_{0}$ and $\tilde{p}=p_{0}$, we obtain

$$
\begin{gathered}
f_{B_{R}}|D v-D w|^{p_{0}} d x \\
\leq c L_{1}^{2} \omega(R)^{2}\left[f_{B_{R}}(|D v|+s)^{p_{2}+\omega(4 R)} \log ^{2}\left(e+(|D v|+s)^{p_{2}+\omega(4 R)}\right) d x\right. \\
\left.\quad+\log ^{2}\left(\frac{1}{R}\right) f_{B_{R}}(|D v|+s)^{p(x)} d x+R^{p_{0}}\right],
\end{gathered}
$$


where $c \equiv c\left(\nu, L, \gamma_{2}\right)$. Next, we use estimates in Orlicz spaces in order to bound the first integral on the right-hand side of the preceding inequality. First, we define averaged $L^{1}$-norm for $f \in L^{1}(\Omega)$ by $\|f\|_{1}:=f_{\Omega}|f| d x$. Using the elementary inequality $\log ^{2}(e+a b) \leq 2\left(\log ^{2}(e+a)+\log ^{2}(e+b)\right)$ for any $a, b \geq 0$ (cf. (32) in [4]) we get

$$
\begin{aligned}
& f_{B_{R}}(|D v|+s)^{p_{2}+\omega(4 R)} \log ^{2}\left(e+(|D v|+s)^{p_{2}+\omega(4 R)}\right) d x \\
& \leq 2 f_{B_{R}}(|D v|+s)^{p_{2}+\omega(4 R)} \log ^{2}\left(e+\frac{(|D v|+s)^{p_{2}+\omega(4 R)}}{\|(|D v|+s)^{p_{2}+\omega(4 R) \|_{1}}}\right) d x \\
& \quad+2 f_{B_{R}}(|D v|+s)^{p_{2}+\omega(4 R)} \log ^{2}\left(e+\left\|(|D v|+s)^{p_{2}+\omega(4 R)}\right\|_{1}\right) d x \\
& =: 2\left(I_{1}+I_{2}\right),
\end{aligned}
$$

with the obvious meaning of $I_{1}$ and $I_{2}$. For the estimate of $I_{1}$ we first use the inequality

$$
f_{\Omega}|f| \log ^{2}\left(e+\frac{|f|}{\|f\|_{1}}\right) d x \leq c(q)\left(f_{\Omega}|f|^{q} d x\right)^{1 / q}, \forall q>1, f \in L \log ^{2} L(\Omega),
$$

with the choice $q:=q\left(n, L / \nu, \gamma_{2}\right):=\left(4+2 \delta_{1}\right) /\left(4+\delta_{1}\right)>1$. Note that this estimate is a consequence of the bound of the Luxemburg norm in the $L \log L$-space in terms of the $L^{q}$ norm (cf. [7, 35, 36], or [4, inequality (28)]). Furthermore, since $q\left(p_{2}+\omega(4 R)\right) \leq$ $q \cdot p_{2}\left(1+\delta_{1} / 4\right)=p_{2}\left(1+\delta_{1} / 2\right)$-recall that $\omega(4 R) \leq \delta_{1} / 4$-we can subsequently use (3.20) to infer that

$I_{1} \leq c\left[f_{B_{R}}(|D v|+s)^{\left(p_{2}+\omega(4 R)\right) \cdot \frac{4+2 \delta_{1}}{4+\delta_{1}}} d x\right]^{\frac{4+\delta_{1}}{4+2 \delta_{1}}} \leq c\left[f_{B_{3 / 2 R}}(|D v|+s)^{p(x)} d x+R^{p_{2}+\omega(4 R)}\right]$,

where $c \equiv c\left(n, \nu, L, \gamma_{2}, M,|\mu|(\Omega)\right)$. For the estimate of $I_{2}$ we first observe from (3.20) (note that $R^{2\left(p_{2}+\omega(4 R)\right)} \leq R^{2 p_{2}}$ ) and (3.15) that

$$
f_{B_{R}}(|D v|+s)^{p_{2}+\omega(4 R)} d x \leq c f_{B_{3 R / 2}}|D v|^{p(x)} d x+c R^{2 p_{2}} \leq c R^{-n-\frac{\alpha}{1-\beta}},
$$

where $\alpha, \beta$ were defined in (3.13) and (3.14) and $c \equiv c\left(n, \nu, L, \gamma_{2}, M,|\mu|(\Omega)\right)$. Here, we have also used $R^{2 p_{2}} \leq R^{-\frac{\alpha}{1-\beta}}$, since $R \leq 1$. Using the preceding estimate twice (note that $\|\cdot\|_{1}$ denotes the averaged $L^{1}$ norm) we obtain the following estimate for $I_{2}$ :

$$
\begin{aligned}
I_{2} & \leq c \log ^{2}\left(e+c R^{-n-\frac{\alpha}{1-\beta}}\right)\left[f_{B_{3 R / 2}}|D v|^{p(x)} d x+R^{2 p_{2}}\right] \\
& \leq c\left[\left(n+\frac{\alpha}{1-\beta}\right) \log \left(\frac{1}{R}\right)+\log (c+\mathrm{e})\right]^{2}\left[f_{B_{3 R / 2}}|D v|^{p(x)} d x+R^{2 p_{2}}\right] \\
& \leq c\left[\log ^{2}\left(\frac{1}{R}\right)+1\right]\left[f_{B_{3 R / 2}}|D v|^{p(x)} d x+R^{2 p_{2}}\right] .
\end{aligned}
$$

At this stage we recall that $\frac{\alpha}{1-\beta} \leq c\left(n, \gamma_{2}\right)$ by the definition of $\alpha, \beta$ and therefore the constant $c$ depends on $n, \nu, L, \gamma_{2}, M,|\mu|(\Omega)$. Furthermore, we have $\log ^{2}\left(\frac{1}{R}\right) R^{p_{2}} \leq e^{-1}$ 
and $\log 1 / R \geq 1$ whenever $R \leq 1 / \mathrm{e}$, which can always be attained by eventually reducing $R_{0}$ such that $R_{0} \leq 1 / \mathrm{e}$. This leads us to

$$
I_{2} \leq c \log ^{2}\left(\frac{1}{R}\right) f_{B_{3 / 2 R}}(|D v|+s)^{p(x)} d x+c R^{p_{0}}
$$

with a constant that depends on $n, \nu, L, \gamma_{2}, M,|\mu|(\Omega)$. Inserting the preceding estimates for $I_{1}$ and $I_{2}$ into (3.23) we deduce the desired estimate for the first term on the right-hand side of (3.22), i.e., we arrive at

$$
f_{B_{R}}|D v-D w|^{p_{0}} d x \leq c L_{1}^{2} \omega(R)^{2}\left[\log ^{2}\left(\frac{1}{R}\right) f_{B_{3 R / 2}}(|D v|+s)^{p(x)} d x+R^{p_{0}}\right],
$$

where $c \equiv c\left(n, \nu, L, \gamma_{2}, M,|\mu|(\Omega)\right)$. This proves the assertion of the lemma.

In the next lemma we combine the comparison estimates from Lemma 3.1 and 3.4 in order to deduce directly a comparison estimate between the original solution $u$ and the solution of the frozen homogeneous problem (3.2) $w$.

Lemma 3.5. Under the assumptions of Theorem 1.1, let $B_{2 R} \equiv B_{2 R}\left(x_{0}\right) \subseteq \Omega$ and $p_{0} \equiv p\left(x_{0}\right)$ and denote by $v$ the solution of (3.1) on $B_{2 R}$ and by $w$ the solution of (3.2) on $B_{R}$. Then there exists a constant $c \equiv c\left(n, \nu, L, \gamma_{2}, M,|\mu|(\Omega)\right)$ and a radius $R_{0} \equiv R_{0}\left(n, L / \nu, L_{1}, \gamma_{2}, \omega(\cdot)\right) \in(0,1]$ such that whenever $0<R \leq R_{0}$ the following estimate holds:

$$
f_{B_{R}}|D u-D w| d x \leq c\left[\frac{|\mu|\left(B_{2 R}\right)}{R^{n-1}}\right]^{\frac{1}{p_{0}-1}}+c\left[L_{1} \omega(R) \log \left(\frac{1}{R}\right)\right]^{\frac{2}{p_{0}}} f_{B_{2 R}}(|D u|+s) d x+c R .
$$

Proof. The proof consists in matching together the two comparison estimates of Lemma 3.1 and 3.4. Thereby it will be necessary to be extremely careful with appearing exponents. In a first step we split the integrand, then use the already available comparison estimates from Lemma 3.1 and 3.4 and finally adapt the appearing exponents. Since at one point we have to pass from the $p(x)$-energy of $v$ to the 1-energy of $u$, we will take advantage of the reverse-type inequality (3.15). In order to adapt the exponents of the radius, we will frequently exploit the localization techniques via the logarithmic continuity of the exponent function $p(\cdot)$ as for instance provided in (2.7). Initially, we let $R_{0} \equiv R_{0}\left(n, L / \nu, \gamma_{2}, \omega(\cdot)\right) \in(0,1]$ be the corresponding radius in Lemma 3.4. Within the proof we shall possibly further reduce this value. Let us start by simply observing that

$$
f_{B_{R}}|D u-D w| d x \leq f_{B_{R}}|D u-D v| d x+f_{B_{R}}|D v-D w| d x=: I_{1}+I_{2}
$$

with the obvious labeling of $I_{1}$ and $I_{2}$.

Applying Lemma 3.1, the expression $I_{1}$ is estimated by

$$
I_{1} \leq c\left[\frac{|\mu|\left(B_{2 R}\right)}{R^{n-1}}\right]^{\frac{1}{p_{0}-1}}+c R
$$


with a constant $c \equiv c\left(n, \nu, L, \gamma_{2}, M,|\mu|(\Omega)\right)$. To treat $I_{2}$, we in turn use Hölder's inequality and Lemma 3.4 to find that

$$
\begin{aligned}
I_{2} & \leq\left[f_{B_{R}}|D v-D w|^{p_{0}}\right]^{\frac{1}{p_{0}}} \\
& \leq c\left[L_{1} \omega(R) \log \frac{1}{R}\right]^{\frac{2}{p_{0}}}\left[f_{B_{3 / 2 R}}(|D v|+s)^{p(x)} d x\right]^{\frac{1}{p_{0}}}+c\left[L_{1} \omega(R)\right]^{\frac{2}{p_{0}}} R,
\end{aligned}
$$

where $c \equiv c\left(n, \nu, L, \gamma_{2},|\mu|(\Omega)\right)$. Now, using inequality (3.15) and taking mean values we find that

$$
\begin{aligned}
& f_{B_{3 / 2 R}}(|D v|+s)^{p(x)} d x \\
& \leq c R^{-n-\frac{\alpha}{1-\beta}+n \frac{\gamma}{1-\beta}}\left[f_{B_{2 R}}(|D u|+s+R) d x+\left[\frac{|\mu|\left(B_{2 R}\right)}{R^{n-1}}\right]^{\frac{1}{p_{0}-1}}\right]^{\frac{\gamma}{1-\beta}} .
\end{aligned}
$$

Recalling the definitions of $\alpha, \beta$ and $\gamma$ in (3.13) and (3.14), a straight forward computation shows that

$$
-n-\frac{\alpha}{1-\beta}+n \frac{\gamma}{1-\beta}=0,
$$

which in turn means that the first radius on the right hand side of the preceding inequality cancels. Our aim is now to "reduce" the exponent $\frac{\gamma}{1-\beta}$ in (3.26) to $p_{0}$, which means to estimate the quantity

$$
J:=\left[f_{B_{2 R}}(|D u|+s+R) d x+\left[\frac{|\mu|\left(B_{2 R}\right)}{R^{n-1}}\right]^{\frac{1}{p_{0}-1}}\right]^{\frac{\gamma}{1-\beta}-p_{0}} .
$$

Therefore, we need to have a closer look at the exponent $\frac{\gamma}{1-\beta}$. For this aim we first consider the reciprocal value $\frac{1-\beta}{\gamma}$. Recalling again the definitions of $\beta$ and $\gamma$, we first see that

$$
\frac{1-\beta}{\gamma}=\frac{p_{1}-p_{2}+\vartheta \frac{p_{2}}{p_{1}}-1}{p_{2}(\vartheta-1)}=\frac{1}{p_{2}}\left[1-\frac{\left(p_{2}-p_{1}\right)\left(1-\frac{\vartheta}{p_{1}}\right)}{\vartheta-1}\right] .
$$

Exploiting (2.6), noting that $1<\vartheta<2$ and hence $\frac{\vartheta}{p_{1}}<1$ we deduce

$$
\frac{1}{p_{2}}\left[1-\frac{\omega(4 R)}{\vartheta-1}\right] \leq \frac{1-\beta}{\gamma} \leq \frac{1}{p_{2}} .
$$

Reducing $R_{0}$ in such a way that we have

$$
\omega\left(4 R_{0}\right) \leq \frac{\vartheta-1}{2}=c(n)
$$

we therefore conclude

$$
\left[1-\frac{\omega(4 R)}{\vartheta-1}\right]^{-1}=\frac{1}{1-\frac{\omega(4 R)}{\vartheta-1}}=1+\frac{\omega(4 R)}{\vartheta-1} \cdot \sum_{k=0}^{\infty}\left[\frac{\omega(4 R)}{\vartheta-1}\right]^{k} \leq 1+2 \frac{\omega(4 R)}{\vartheta-1},
$$

and therefore arrive at

$$
p_{2} \leq \frac{\gamma}{1-\beta} \leq p_{2}\left[1+2 \frac{\omega(4 R)}{\vartheta-1}\right]
$$


This shows us that

$$
0 \leq \frac{\gamma}{1-\beta}-p_{0} \leq \omega(4 R)\left[1+\frac{2 \gamma_{2}}{\vartheta-1}\right] \leq c\left(n, \gamma_{2}\right)
$$

With this information we can bound $J$ as follows:

$$
\begin{aligned}
J & \leq c\left(n, \gamma_{2}, M,|\mu|(\Omega)\right) R^{-n\left(\frac{\gamma}{1-\beta}-p_{0}\right)} \\
& \leq c\left(n, \gamma_{2}, M,|\mu|(\Omega)\right) R^{-\omega(4 R) \cdot n\left(1+\frac{2 \gamma_{2}}{\vartheta-1}\right)} \leq c\left(n, L, \gamma_{2}, M,|\mu|(\Omega)\right),
\end{aligned}
$$

where we have used (2.7) in the final estimate. Joining the preceding estimate with (3.26) we arrive at

$$
f_{B_{3 / 2 R}}(|D v|+s)^{p(x)} d x \leq c\left[f_{B_{2 R}}(|D u|+s) d x+\left[\frac{|\mu|\left(B_{2 R}\right)}{R^{n-1}}\right]^{\frac{1}{p_{0}-1}}+R\right]^{p_{0}},
$$

which in combination with (3.25) yields that

$$
I_{2} \leq c\left[L_{1} \omega(R) \log \frac{1}{R}\right]^{\frac{2}{p_{0}}}\left[f_{B_{2 R}}(|D u|+s) d x+\left[\frac{|\mu|\left(B_{2 R}\right)}{R^{n-1}}\right]^{\frac{1}{p_{0}-1}}+R\right],
$$

where $c \equiv c\left(n, \nu, L, \gamma_{2}, M,|\mu|(\Omega)\right)$. We now combine the preceding estimate for $I_{2}$ with the one for $I_{1}$ in (3.24) and reduce $R_{0}$ in such a way that $L_{1} \omega\left(R_{0}\right) \log \frac{1}{R_{0}} \leq 1$ holds, which is possible thanks to (1.9). Then $R_{0}$ additionally depends on $L_{1}$. This finally yields the desired comparison estimate.

3.4. Decay below the natural growth exponent. Here, we shall merge together our comparison estimate from Lemma 3.5 with the decay estimate from Theorem 2.2 which is satisfied by the solution $w$ of the frozen coefficient equation. This will lead us to the following $L^{1}$-decay estimate for the solution $u$ of the original problem:

Lemma 3.6. Under the assumptions of Theorem 1.1, there exist constants $\beta \equiv$ $\beta\left(n, L / \nu, L_{1}, \gamma_{2}\right) \in(0,1]$ and $\left.c_{1} \equiv c_{1}\left(n, \nu, L, \gamma_{2}, M,|\mu|(\Omega)\right)\right) \geq 1$ and a radius $R_{0} \equiv$ $R_{0}\left(n, L / \nu, L_{1}, \gamma_{2}, \omega(\cdot)\right) \in(0,1]$ such that the following holds: whenever $B_{\varrho} \subseteq B_{R} \subset$ $B_{2 R} \equiv B_{2 R}\left(x_{0}\right) \subseteq \Omega$ are concentric balls with $R \leq R_{0}$ and $p_{0} \equiv p\left(x_{0}\right)$, then the following estimate holds:

$$
\begin{aligned}
& f_{B_{\varrho}}\left|D u-(D u)_{B_{\varrho}}\right| d x \\
& \leq c_{1}\left[\left(\frac{\varrho}{R}\right)^{\beta}+\left(\frac{R}{\varrho}\right)^{n}\left[L_{1} \omega(R) \log \frac{1}{R}\right]^{\frac{2}{p_{0}}}\right] f_{B_{2 R}}\left|D u-(D u)_{B_{2 R}}\right| d x \\
& \quad+c_{1}\left(\frac{R}{\varrho}\right)^{n}\left[\left[\frac{|\mu|\left(B_{2 R}\right)}{R^{n-1}}\right]^{\frac{1}{p_{0}-1}}+\left[L_{1} \omega(R) \log \frac{1}{R}\right]^{\frac{2}{p_{0}}}\left(\left|(D u)_{B_{2 R}}\right|+s\right)+R\right] .
\end{aligned}
$$

Proof. For a ball $B_{2 R}$ as in the statement of the lemma we define the comparison function $w$ on $B_{R}$ according to (3.2). Since the function $w$ solves a homogeneous equation with frozen coefficients of the form (3.2), we may apply Theorem 2.2 to conclude that there exist constants $\beta \in(0,1]$ and $c \geq 1$, both depending only on $n, \nu, L, \gamma_{2}$-see Remark 2.3 - such that the decay estimate

$$
f_{B_{\varrho}}\left|D w-(D w)_{B_{\varrho}}\right| d x \leq c\left(\frac{\varrho}{R}\right)^{\beta} f_{B_{R}}\left|D w-(D w)_{B_{R}}\right| d x
$$


holds for any $\varrho<R$. Carrying this estimate over to $u$ yields

$$
\begin{aligned}
f_{B_{\varrho}}\left|D u-(D u)_{B_{\varrho}}\right| d x & \leq f_{B_{\varrho}}\left|D w-(D w)_{B_{\varrho}}\right| d x+2 f_{B_{\varrho}}|D u-D w| d x \\
& \leq c\left(\frac{\varrho}{R}\right)^{\beta} f_{B_{R}}\left|D w-(D w)_{B_{R}}\right| d x+2\left(\frac{R}{\varrho}\right)^{n} f_{B_{R}}|D u-D w| d x \\
& \leq c\left(\frac{\varrho}{R}\right)^{\beta} f_{B_{R}}\left|D u-(D u)_{B_{R}}\right| d x+c\left(\frac{R}{\varrho}\right)^{n} f_{B_{R}}|D u-D w| d x
\end{aligned}
$$

where $c \equiv c(n, \nu, L, \gamma)$. The first integral appearing on the right-hand side of the preceding inequality is further estimated as follows:

$$
f_{B_{R}}\left|D u-(D u)_{B_{R}}\right| d x \leq 2 f_{B_{R}}\left|D u-(D u)_{B_{2 R}}\right| d x \leq 2^{n+1} f_{B_{2 R}}\left|D u-(D u)_{B_{2 R}}\right| d x,
$$

whereas for the second one we shall apply Lemma 3.5. At this stage we let $R_{0} \equiv$ $R_{0}\left(n, L / \nu, L_{1}, \gamma_{2}, \omega(\cdot)\right)$ denote the radius from Lemma 3.5 and suppose that $R \leq R_{0}$. Then, the application of the lemma yields

$$
f_{B_{R}}|D u-D w| d x \leq c\left[L_{1} \omega(R) \log \frac{1}{R}\right]^{\frac{2}{p_{0}}} f_{B_{2 R}}(|D u|+s) d x+c\left[\frac{|\mu|\left(B_{2 R}\right)}{R^{n-1}}\right]^{\frac{1}{p_{0}-1}}+c R
$$

where $c \equiv c\left(n, \nu, L, \gamma_{2}, M,|\mu|(\Omega)\right)$. Finally, we estimate the first integral of the righthand side by

$$
f_{B_{2 R}}(|D u|+s) d x \leq f_{B_{2 R}}\left|D u-(D u)_{B_{2 R}}\right| d x+\left|(D u)_{B_{2 R}}\right|+s .
$$

Merging together the preceding estimates yields the assertion of the lemma.

3.5. Proof of Theorem 1.1. We now have the prerequisites to prove the first main theorem of the paper which will be accomplished in four steps.

Step 1: Decay estimate. Let $\beta \equiv \beta\left(n, L / \nu, L_{1}, \gamma_{2}\right) \in(0,1]$ and $c_{1} \equiv c_{1}\left(n, \nu, L, \gamma_{2}\right.$, $M,|\mu|(\Omega)) \geq 1$ be the corresponding constants from Lemma 3.6 and $R_{0} \equiv R_{0}(n, L / \nu$, $\left.L_{1}, \gamma_{2}, \omega(\cdot)\right) \in(0,1]$ the corresponding radius. Note that in the course of the proof we shall possibly further reduce $R_{0}$. Next, we define $H \in \mathbf{R}$ by

$$
c_{1}\left(\frac{1}{H}\right)^{\beta}=\frac{1}{4} \text {. }
$$

Note that this implies $H \geq 2$ depending only on $\left.n, \nu, L, \gamma_{2}, M,|\mu|(\Omega)\right)$. With this choice we start reducing the value of $R_{0}$ in order to have

$$
c_{1} H^{n}\left[L_{1} \omega\left(R_{0}\right) \log \frac{1}{R_{0}}\right]^{\frac{2}{\gamma_{2}}} \leq \frac{1}{4},
$$

which is possible due to (1.9). Note that $R_{0}$ now depends on $n, \nu, L, L_{1}, \gamma_{2}, M,|\mu|(\Omega)$, $\omega(\cdot)$. Moreover, note that condition (3.30) together with $L_{1} \omega(\tilde{R}) \log \frac{1}{\tilde{R}} \leq 1$ and $p_{0} \leq \gamma_{2}$ particularly implies

$$
c_{1} H^{n}\left[L_{1} \omega\left(R_{0}\right) \log \frac{1}{R_{0}}\right]^{\frac{2}{p_{0}}} \leq \frac{1}{4} .
$$


Applying Lemma 3.6 on the concentric balls $B_{R / H} \subseteq B_{R / 2} \subset B_{R} \subseteq \Omega$ with $R \leq R_{0}$ and keeping in mind the previous choices and the fact that $\omega(\cdot)$ is nondecreasing yields

$$
\begin{aligned}
f_{B_{R / H}} \mid & D u-(D u)_{B_{R / H}}\left|d x \leq \frac{1}{2} f_{B_{R}}\right| D u-(D u)_{B_{R}} \mid d x \\
& +c_{1} H^{n}\left[\left[\frac{|\mu|\left(B_{R}\right)}{R^{n-1}}\right]^{\frac{1}{p_{0}-1}}+\left[L_{1} \omega(R) \log \frac{1}{R}\right]^{\frac{2}{p_{0}}}\left(\left|(D u)_{B_{R}}\right|+s\right)+R\right] .
\end{aligned}
$$

Step 2: Dyadic sequences. We now fix a ball $B_{2 R}\left(x_{0}\right) \subseteq \Omega$ as in the statement of Theorem 1.1. For $i \in \mathbf{N}_{0}$ we define

$$
B_{i}:=B_{R_{i}}\left(x_{0}\right), \quad \text { where } R_{i}:=R / H^{i}
$$

and

$$
k_{i}:=\left|(D u)_{B_{i}}\right|
$$

Then, for any $\ell \in \mathbf{N}$ we have

$$
\begin{aligned}
k_{\ell+1}=\sum_{i=0}^{\ell}\left(k_{i+1}-k_{i}\right)+k_{0} & \leq \sum_{i=0}^{\ell} f_{B_{i+1}}\left|D u-(D u)_{B_{i}}\right| d x+k_{0} \\
& \leq \sum_{i=0}^{\ell} H^{n} f_{B_{i}}\left|D u-(D u)_{B_{i}}\right| d x+k_{0} .
\end{aligned}
$$

Setting

$$
A_{i}:=f_{B_{i}}\left|D u-(D u)_{B_{i}}\right| d x
$$

the preceding inequality can be rewritten as

$$
k_{\ell+1} \leq H^{n} \sum_{i=0}^{\ell} A_{i}+k_{0} .
$$

Now, we observe that we can use (3.31) with $R=R_{i-1}$ for any $i \geq 1$ which, with the previous notations, reads as

$$
A_{i} \leq \frac{1}{2} A_{i-1}+c\left[\frac{|\mu|\left(B_{i-1}\right)}{R_{i-1}^{n-1}}\right]^{\frac{1}{p_{0}-1}}+c\left[L_{1} \omega\left(R_{i-1}\right) \log \frac{1}{R_{i-1}}\right]^{\frac{2}{p_{0}}}\left(k_{i-1}+s\right)+c R_{i-1},
$$

with $\left.c \equiv c\left(n, \nu, L, \gamma_{2}, M,|\mu|(\Omega)\right)\right)$, where we have taken into account that $H$ only depends on $n, \nu, L, \gamma_{2}$. Summing up the preceding inequality for $i=1, \ldots, \ell$ we obtain

$$
\sum_{i=1}^{\ell} A_{i} \leq \frac{1}{2} \sum_{i=1}^{\ell-1} A_{i-1}+c \sum_{i=0}^{\ell-1}\left[\left[\frac{|\mu|\left(B_{i}\right)}{R_{i}^{n-1}}\right]^{\frac{1}{p_{0}-1}}+\left[L_{1} \omega\left(R_{i}\right) \log \frac{1}{R_{i}}\right]^{\frac{2}{p_{0}}}\left(k_{i}+s\right)+R_{i}\right],
$$

which after absorbing $\frac{1}{2} \sum_{i=1}^{\ell-1} A_{i-1}$ on the left-hand side yields

$$
\sum_{i=1}^{\ell} A_{i} \leq A_{0}+c \sum_{i=0}^{\ell-1}\left[\left[\frac{|\mu|\left(B_{i}\right)}{R_{i}^{n-1}}\right]^{\frac{1}{p_{0}-1}}+\left[L_{1} \omega\left(R_{i}\right) \log \frac{1}{R_{i}}\right]^{\frac{2}{p_{0}}}\left(k_{i}+s\right)+R_{i}\right] .
$$


We now use the preceding inequality to estimate the right-hand side of (3.35) yielding for any $\ell \geq 1$ that

$$
k_{\ell+1} \leq k_{0}+c A_{0}+c \sum_{i=0}^{\ell-1}\left[\left[\frac{|\mu|\left(B_{i}\right)}{R_{i}^{n-1}}\right]^{\frac{1}{p_{0}-1}}+\left[L_{1} \omega\left(R_{i}\right) \log \frac{1}{R_{i}}\right]^{\frac{2}{p_{0}}}\left(k_{i}+s\right)+R_{i}\right] .
$$

where $c \equiv c\left(n, \nu, L, \gamma_{2}, M,|\mu|(\Omega)\right)$ and we again have taken into account that $H$ depends on $n, \nu, L, \gamma_{2}$.

Step 3: Uniform bounds and Wolff potentials. Here, we shall find bounds independent of $\ell$ for the sums on the right-hand side of (3.37). Let us start with the first one involving the measure $\mu$. Here, we proceed as in the proof of estimate (3.56) in [16] to infer that

$$
\sum_{i=0}^{\ell-1}\left[\frac{|\mu|\left(B_{i}\right)}{R_{i}^{n-1}}\right]^{\frac{1}{p_{0}-1}} \leq c \mathbf{W}_{\frac{1}{p(\cdot)}, p(\cdot)}^{\mu}\left(x_{0}, 2 R\right), \quad \text { for any } \ell \geq 1,
$$

where $c \equiv \frac{2^{n-1}}{\log 2}+\frac{H^{n-1}}{\log H}$ and therefore $c$ depends on $n, \nu, L, \gamma_{2}, M,|\mu|(\Omega)$. Next, we shall consider the sum in (3.37) involving the modulus $\omega(\cdot)$ and we will show that

$$
\sum_{i=0}^{\ell-1}\left[\omega\left(R_{i}\right) \log \frac{1}{R_{i}}\right]^{\frac{2}{p_{0}}} \leq 2 d\left(x_{0}, 2 R\right),
$$

where $d(\cdot)$ has been defined in (1.9). Proceeding similarly to the last estimate, also using that $\omega(\cdot)$ is non-decreasing, we obtain

$$
\begin{aligned}
\sum_{i=0}^{\ell-1}\left[\omega\left(R_{i}\right) \log \frac{1}{R_{i}}\right]^{\frac{2}{p_{0}}} & \leq \sum_{i=0}^{\infty}\left[\omega\left(R_{i}\right) \log \frac{1}{R_{i}}\right]^{\frac{2}{p_{0}}} \\
& \leq\left[\omega(R) \log \frac{1}{R}\right]^{\frac{2}{p_{0}}}+\sum_{i=0}^{\infty}\left[\omega\left(R_{i+1}\right) \log \frac{1}{R_{i+1}}\right]^{\frac{2}{p_{0}}} \\
& \leq \frac{1}{\log 2} \int_{R}^{2 R}\left[\omega(\varrho) \log \frac{1}{\varrho}\right]^{\frac{2}{p_{0}}} \frac{d \varrho}{\varrho}+\frac{1}{\log H} \sum_{i=0}^{\infty} \int_{R_{i+1}}^{R_{i}}\left[\omega(\varrho) \log \frac{1}{\varrho}\right]^{\frac{2}{p_{0}}} \frac{d \varrho}{\varrho} \\
& \leq \frac{1}{\log 2} \int_{0}^{2 R}\left[\omega(\varrho) \log \frac{1}{\varrho}\right]^{\frac{2}{p_{0}}} \frac{d \varrho}{\varrho},
\end{aligned}
$$

where in the last line we have taken into account that $(\log H)^{-1} \leq(\log 2)^{-1}$ since $H \geq 2$. At this stage (3.39) follows from the very definition of $d(\cdot)$ in (1.9) and the fact that $\log 2 \geq 1 / 2$. For later use we further restrict $R_{0}$ in order to have

$$
d\left(x_{0}, 2 R_{0}\right) \leq \frac{1}{L_{1}} \leq \frac{1}{L_{1}^{2 / p_{0}}},
$$

which is possible thanks to (1.9) and the facts that $L_{1} \geq 1$ and $p_{0} \geq 2$. Note that $R_{0}$ now additionally depends on $p_{0}$, i.e., it is of the form $R_{0} \equiv R_{0}\left(n, \nu, L, L_{1}, \gamma_{2}, M,|\mu|(\Omega)\right.$, $\left.\omega(\cdot), p_{0}\right)$. Finally, we estimate the third sum in (3.37) as follows:

$$
\sum_{i=0}^{\ell-1} R_{i}=R \sum_{i=0}^{\ell-1} H^{-i} \leq 2 R
$$


where we have taken into account that $H \geq 2$. Joining (3.38)-(3.41) with (3.37) and taking into account that

$$
k_{0}+A_{0}+s \leq c f_{B_{R}\left(x_{0}\right)}(|D u|+s) d x
$$

we arrive at

$$
k_{\ell+1} \leq c_{2} \mathscr{M}+c_{3} L_{1}^{\frac{2}{p_{0}}} \sum_{i=0}^{\ell-1}\left[\omega\left(R_{i}\right) \log \frac{1}{R_{i}}\right]^{\frac{2}{p_{0}}} k_{i}, \quad \text { for every } \ell \geq 1,
$$

where $c_{2}, c_{3} \geq 1$ depend on $n, \nu, L, \gamma_{2}, M,|\mu|(\Omega)$ and we have set

$$
\mathscr{M}:=f_{B_{R}\left(x_{0}\right)}(|D u|+s) d x+\mathbf{W}_{\frac{1}{p(\cdot)}, p(\cdot)}^{\mu}\left(x_{0}, 2 R\right)+R .
$$

Finally, to complete (3.42) also for $\ell=-1,0$ we estimate

$$
k_{0}+k_{1} \leq\left(1+H^{n}\right) f_{B_{R}\left(x_{0}\right)}|D u| d x \leq c_{2} \mathscr{M},
$$

which can be obtained by eventually enlarging the constant $c_{2}$ without changing the dependencies.

Step 4: Induction and final conclusion. Here, we restrict the value of $R_{0}$ for the last time in such a way that

$$
d\left(x_{0}, 2 R_{0}\right) \leq \frac{1}{4 c_{3} L_{1}},
$$

holds, where $c_{3}$ denotes the corresponding constant appearing in (3.42). This finally determines $R_{0}$ as a positive constant satisfying (3.30), (3.40) and (3.45) and therefore depending on $n, \nu, L, L_{1}, \gamma_{2}, M,|\mu|(\Omega), \omega(\cdot)$ and $p_{0}$. We will now prove by induction that

$$
k_{\ell} \leq 2 c_{2} \mathscr{M} \quad \text { for any } \ell \in \mathbf{N}_{0},
$$

where $c_{2}$ and $\mathscr{M}$ have been defined in (3.42) and (3.43). For $\ell=0,1$ our assertion (3.46) has already been shown in (3.44). Now, we assume (3.46) holds for any $\ell \leq m$, with some $m \geq 1$ and prove it for $m+1$. Using (3.42), (3.46), (3.39), (3.45) and $L_{1}^{\frac{2}{p_{0}}} \leq L_{1}$ we get

$$
\begin{aligned}
k_{m+1} & \leq c_{2} \mathscr{M}+c_{3} L_{1}^{\frac{2}{p_{0}}} \sum_{i=0}^{m-1}\left[\omega\left(R_{i}\right) \log \frac{1}{R_{i}}\right]^{\frac{2}{p_{0}}} k_{i} \\
& \leq c_{2} \mathscr{M}+2 c_{3} c_{2} \mathscr{M} L_{1} \sum_{i=0}^{m-1}\left[\omega\left(R_{i}\right) \log \frac{1}{R_{i}}\right]^{\frac{2}{p_{0}}} \\
& \leq c_{2} \mathscr{M}+4 c_{3} c_{2} \mathscr{M} L_{1} d\left(x_{0}, 2 R\right) \\
& \leq c_{2} \mathscr{M}+c_{2} \mathscr{M}=2 c_{2} \mathscr{M}
\end{aligned}
$$

proving our assertion (3.46).

At this stage we recall that we assumed $D u$ to be continuous and therefore we may conclude that for any $x_{0} \in \Omega$ there holds

$$
\left|D u\left(x_{0}\right)\right|=\lim _{\ell \rightarrow \infty} k_{\ell} \leq 2 c_{2} \mathscr{M} .
$$


Together with the definition of $\mathscr{M}$ in (3.43) we now conclude the desired estimate (1.10). Finally, we observe that (3.40) and (3.45) were the only conditions on $R_{0}$ causing its dependence upon $p\left(x_{0}\right)$. Therefore, assuming (1.6) instead of (1.9) and replacing $d\left(x_{0}, 2 R_{0}\right)$ by $d\left(2 R_{0}\right)$ leads us to a constant $R_{0}$ independent of $p\left(x_{0}\right)$. This completes the proof of Theorem 1.1.

Remark 3.7. Note that the only point in the proof where we needed the assumption $u \in C^{1}(\Omega)$ is the passage to the limit in (3.47). For the rest of the proof it we could also have worked with a solution $u \in W_{\text {loc }}^{1, p(\cdot)}(\Omega)$. Indeed, we can repeat the proof line by line for such a solution, but taking into account that the convergence in (3.47) eventually only takes place in Lebesgue points of $D u$. Therefore, we end up with (1.10) for almost every $x_{0} \in \Omega$.

\section{Gradient estimate for general solutions}

In this Chapter we consider Dirichlet problems involving a right-hand side measure $\mu$ and thereby prove the applications of our a priori estimate from Theorem 1.1 provided in Theorems 1.3 and 1.4.

4.1. SOLAs and proof of Theorem 1.4. In the case where $\mu$ does not belong to $W^{-1, p^{\prime}(\cdot)}(\Omega)$ the general existence theory of weak solutions does not apply and therefore one has to consider a more general notion of solution. In the literature there are different approaches, see [8, 40, 49]. We shall follow the one introduced in $[9,10,14]$ for the constant exponent case. More precisely, we shall consider a SOLA (Solution Obtained by Limit of Approximations) which is a function $u \in W^{1, q(\cdot)}(\Omega)$, with $q(\cdot)<\bar{q}(\cdot):=\min \{n(p(\cdot)-1) /(n-1), p(\cdot)\}$ satisfying (1.11) in the weak sense and which is found by means of approximations, i.e., it is obtainable as pointwise limit of weak solutions $\left(W_{0}^{1, p(\cdot)}(\Omega)\right.$-solutions). Note that $u \in W^{1, q(\cdot)}(\Omega)$ for any $q(\cdot)<\bar{q}(\cdot)$ implies $u \in W^{1, p(\cdot)-1}(\Omega)$ and therefore the weak formulation of $(1.11)$ makes sense for a SOLA. Moreover, in the case that $\mu \in L^{1}(\Omega)$ the SOLA is unique, in the class of such solutions. Note that there is in general no uniqueness in $W^{1, q(\cdot)}$; see the counterexamples already found for the constant coefficient case in [50, 46]. Concerning the existence of a SOLA we note that, since we are assuming $p(\cdot) \geq 2$, in particular cases (for instance when $n \leq 10$, or $p(\cdot) \equiv$ const) the entropy solution constructed in [49, Theorem 1.3] or [8, Theorem 2.1] coincides with the SOLA (see [49, Remark 5.7]), and hence we can infer existence from there. However, in our case of exponents $p(\cdot) \geq 2$ lying above the critical exponent $2-1 / n$ the existence theory is essentially easier and we can infer existence - and uniqueness when $\mu \in L^{1}(\Omega)$ - of a SOLA in the case of coefficients with non standard growth in the spirit of $[9,10,14]$ via the methods of $[8,40,49]$. For the sake of briefness we shall not give the whole proof here, but only the main steps and the approximation procedure leading to the gradient estimate (1.10) for a SOLA.

We consider a Radon measure $\mu$ defined on $\Omega$ with finite total mass. Extending $\mu$ on $\mathbf{R}^{n} \backslash \Omega$ by zero we may assume that $\mu$ is defined on $\mathbf{R}^{n}$. Now, let $\phi \in C_{0}^{\infty}\left(B_{1}\right)$ be a standard symmetric and non-negative mollifier with $\|\phi\|_{L^{1}\left(\mathbf{R}^{n}\right)}=1$. For any $h \in \mathbf{N}$ we define $\phi_{h}(x):=h^{n} \phi(h x)$, for $x \in \mathbf{R}^{n}$ and

$$
\mu_{h}(x):=\left(\mu * \phi_{h}\right)(x), \quad x \in \Omega .
$$


Then, by standard properties of convolutions we know that the function $\mu_{h}: \mathbf{R}^{n} \rightarrow \mathbf{R}$ is smooth, particularly $\mu_{h} \in W^{-1, p^{\prime}(\cdot)}(\Omega)$ and therefore there exists a unique weak solution $u_{h} \in W_{0}^{1, p(\cdot)}(\Omega)$ of the Dirichlet problem

$$
\begin{cases}-\operatorname{div} a\left(x, D u_{h}\right)=\mu_{h} & \text { in } \Omega \\ u_{h}=0 & \text { on } \partial \Omega .\end{cases}
$$

By the construction of $\mu_{h}$ we know that $\mu_{h} \rightarrow \mu$ weakly in the sense of measures as $h \rightarrow \infty$ and $\left\|\mu_{h}\right\|_{L^{1}\left(\mathbf{R}^{n}\right)} \leq|\mu|(\Omega)$. The following lemma will provide a uniform bound for $u_{h}$ in a suitable Sobolev space.

Lemma 4.1. Let $h \in \mathbf{N}$ and $u_{h} \in W_{0}^{1, p(\cdot)}(\Omega)$ be a weak solution to (4.2) as above, particularly with $\mu_{h} \in L^{1}(\Omega) \cap W^{-1, p^{\prime}(\cdot)}(\Omega)$ and let

$$
1 \leq q(\cdot)<\bar{q}(\cdot) \equiv \min \left\{\frac{n(p(\cdot)-1)}{n-1}, p(\cdot)\right\} .
$$

Then, there exists a constant $c \equiv c\left(n, \nu, \gamma_{2}, \Omega,|\mu|(\Omega), q(\cdot)\right)$-independent of $h$-such that

$$
\int_{\Omega}\left|D u_{h}\right|^{q(x)} d x \leq c .
$$

Proof. For $k \in \mathbf{N}$ we set $D_{k}:=\left\{x \in \Omega:\left|u_{h}(x)\right| \leq k\right\}$. Similarly to the proof of Lemma 3.1, i.e., by testing the weak formulation of (4.2) with the truncation $\varphi:=T_{k}\left(u_{h}\right)$ we can show that

$$
\int_{D_{k}}\left|D u_{h}\right|^{p(x)} d x \leq c\left(\nu, \gamma_{2}\right) k\left\|\mu_{h}\right\|_{L^{1}(\Omega)} \leq c\left(\nu, \gamma_{2}\right) k|\mu|(\Omega),
$$

and similarly, testing with $\varphi:=\Phi_{k}\left(u_{h}\right)$ yields

$$
\int_{C_{k}}\left|D u_{h}\right|^{p(x)} d x \leq c\left(\nu, \gamma_{2}\right)\left\|\mu_{h}\right\|_{L^{1}(\Omega)} \leq c\left(\nu, \gamma_{2}\right)|\mu|(\Omega),
$$

where we have set $C_{k}:=\left\{x \in \Omega: k<\left|u_{h}(x)\right| \leq k+1\right\}$. At this stage the assertion of the lemma follows from [8, Lemma 2.1]. Note that the restriction $p(\cdot)<n$ can be avoided by a suitable choice of the exponents in the Sobolev inequality: when $p^{-} \geq n$ (with the notation from [8]) one replaces $q^{+*}$ by $\alpha$, where $\alpha$ is taken in such a way that $\frac{q^{+}}{p^{-}-q^{+}}<\alpha<\frac{p^{-} q^{+}}{p^{-}-q^{+}}$.

From Lemma 4.1 we infer that $\left(u_{h}\right)_{h \in \mathbf{N}}$ is uniformly bounded in $W_{0}^{1, q(\cdot)}(\Omega)$ for any $q(\cdot)$ as in (4.3). Therefore, we can find a-not relabeled-subsequence and a function $u \in W_{0}^{1, q(\cdot)}(\Omega)$ such that

$$
\begin{cases}u_{h} \rightarrow u & \text { weakly in } W_{0}^{1, q(\cdot)}(\Omega), \\ u_{h} \rightarrow u & \text { strongly in } L^{q(\cdot)}(\Omega), \\ u_{h} \rightarrow u & \text { a.e. in } \Omega .\end{cases}
$$

Note that this is not yet sufficient in order to pass to the limit in (4.2). However, with similar arguments as in [9] we can show that - up to a further not relabeled subsequence - we have the strong convergence

$$
D u_{h} \rightarrow D u \text { strongly in } L^{q(\cdot)}(\Omega) \text { for every } q(\cdot) \leq \bar{q}(\cdot) \text { and a.e. in } \Omega \text {. }
$$


This allows to pass to the limit in (4.2) and to infer that $u$ is a SOLA of (1.11). Thereby, the notion of a SOLA is justified since $u$ indeed is a pointwise limit of the more regular solutions $\left(u_{h}\right)$. Moreover, by arguments similar to [14] one can show that the SOLA is unique when $\mu \in L^{1}(\Omega)$, i.e., $u$ is the only solution to (1.11) obtainable as a pointwise limit of $W_{0}^{1, p(\cdot)}$-solutions. Now, we come to the

Proof of Theorem 1.4. We let $\mu_{h}$ and $u_{h}$, with $h \in \mathbf{N}$ be as in (4.1) and (4.2). Then, since $u_{h} \in W_{0}^{1, p(\cdot)}(\Omega)$, by Remark 3.7 we can apply estimate (3.37) to $u_{h}$, which then reads as follows:

$k_{\ell+1}^{(h)} \leq k_{0}^{(h)}+c A_{0}^{(h)}+c \sum_{i=0}^{\ell-1}\left[\left[\frac{\left|\mu_{h}\right|\left(B_{i}\right)}{R_{i}^{n-1}}\right]^{\frac{1}{p_{0}-1}}+c\left[L_{1} \omega\left(R_{i}\right) \log \frac{1}{R_{i}}\right]^{\frac{2}{p_{0}}}\left(k_{i}^{(h)}+s\right)+c R_{i}\right]$,

where we have set (in accordance with (3.33) and (3.34)):

$$
k_{i}^{(h)}:=\left|\left(D u_{h}\right)_{B_{i}}\right| \quad \text { and } \quad A_{0}^{(h)}:=f_{B_{R}}\left|D u_{h}-\left(D u_{h}\right)_{B_{R}}\right| d x .
$$

Note that under condition (1.6) it is possible to prove that $D u \in C^{0}\left(\Omega, \mathbf{R}^{n}\right)$. Passing to the limit $h \rightarrow \infty$ in the preceding inequality - which is justified by (4.4) and the fact that $\mu_{h} \rightarrow \mu$ weakly in measure -yields

$$
k_{\ell+1} \leq k_{0}+c A_{0}+c \sum_{i=0}^{\ell-1}\left[\left[\frac{|\mu|\left(\bar{B}_{i}\right)}{R_{i}^{n-1}}\right]^{\frac{1}{p_{0}-1}}+c\left[L_{1} \omega\left(R_{i}\right) \log \frac{1}{R_{i}}\right]^{\frac{2}{p_{0}}}\left(k_{i}+s\right)+c R_{i}\right],
$$

with the notation from (3.33) and (3.34). At this stage the proof is essentially the same as the one of Theorem 1.1 after (3.37), but taking into account that the convergence now only takes place in the Lebesgue points of $D u$, and therefore almost everywhere. Moreover, in order to deal with the presence of $|\mu|\left(\bar{B}_{i}\right)$ rather than $|\mu|\left(B_{i}\right)$ in the previous estimate, the balls have to be slightly enlarged when estimating the sum in terms of the Wolff potential $\mathbf{W}_{\frac{1}{p(\cdot)}, p(\cdot)}^{\mu}\left(x_{0}, 2 R\right)$ in the analogue of (3.38). But this is always possible without difficulties since we already took the Wolff potential on the larger ball $B_{2 R}$. Finally, the asserted $L^{\infty}$ estimate follows from (1.10) by a covering argument and taking into account the remark in Theorem 1.1 stating that under the hypothesis (1.6) instead of (1.9) the radius $R_{0}$ is independent of $p\left(x_{0}\right)$. This finishes the proof of Theorem 1.4

4.2. Weak solutions and proof of Theorem 1.3. Here, we are dealing within the setting of Theorem 1.3 where $\mu$ already belongs to the dual space $W^{-1, p^{\prime}(\cdot)}(\Omega)$ and therefore the solution is a weak one in the usual sense.

Proof of Theorem 1.3. The present proof, where we may assume $\mu \in W^{-1, p^{\prime}(\cdot)}(\Omega)$, is essentially easier as the one of Theorem 1.4 since now $u \in W^{1, p(\cdot)}(\Omega)$ is a weak solution of (1.2) in the usual sense. Here, we define the local approximations $u_{h} \in$ $W^{1, p(\cdot)}\left(\Omega^{\prime}\right)$ by

$$
\begin{cases}-\operatorname{div} a\left(x, D u_{h}\right)=\mu_{h} & \text { in } \Omega^{\prime} \\ u_{h}=u & \text { on } \partial \Omega^{\prime}\end{cases}
$$

whenever $\Omega^{\prime} \Subset \Omega$ and where the mollification $\mu_{h} \equiv \mu * \phi_{h}$ for $h \in \mathbf{N}$ is defined as in (4.1); particularly we now have $\mu_{h} \rightarrow \mu$ in $W^{-1, p^{\prime}(\cdot)}\left(\Omega^{\prime}\right)$. Let us now show that

$$
u_{h} \rightarrow u \text { strongly in } W^{1, p(\cdot)}\left(\Omega^{\prime}\right) \text {. }
$$


From the weak formulations of (1.2) and (4.5) - where we use the test function $u_{h}-$ $u$-and the monotonicity $(2.2)$ of the vector field $a(\cdot)$ we infer

$$
\begin{aligned}
\frac{\nu}{c\left(\gamma_{2}\right)} \int_{\Omega^{\prime}}\left|D u_{h}-D u\right|^{p(x)} d x & \leq \int_{\Omega^{\prime}}\left\langle a\left(x, D u_{h}\right)-a(x, D u), D u_{h}-D u\right\rangle d x \\
& =\int_{\Omega^{\prime}}\left\langle\mu_{h}-\mu, u_{h}-u\right\rangle_{W^{-1, p^{\prime}(\cdot)} \times W^{1, p(\cdot)}} d x .
\end{aligned}
$$

where $\langle\cdot, \cdot\rangle$ denotes the duality pairing between $W^{-1, p^{\prime}(\cdot)}$ and $W^{1, p(\cdot)}$. From the previous inequality we conclude that

$$
\int_{\Omega^{\prime}}\left|D u_{h}-D u\right|^{p(x)} d x \leq \frac{c\left(\gamma_{2}\right)}{\nu}\left\|u_{h}-u\right\|_{W^{1, p(\cdot)}\left(\Omega^{\prime}\right)}\left\|\mu_{h}-\mu\right\|_{W^{-1, p^{\prime}(\cdot)}\left(\Omega^{\prime}\right)},
$$

which together with the fact that

$$
\int_{\Omega^{\prime}}\left|D u_{h}-D u\right|^{p(x)} d x \geq \min \left\{\left\|D u_{h}-D u\right\|_{L^{p(\cdot)}\left(\Omega^{\prime}\right)}^{2},\left\|D u_{h}-D u\right\|_{L^{p(\cdot)}\left(\Omega^{\prime}\right)}^{\gamma_{2}}\right\} .
$$

Note that $2 \leq p(\cdot) \leq \gamma_{2}$ and Poincaré's inequality yields that

$$
\begin{aligned}
& \min \left\{\left\|u_{h}-u\right\|_{W^{1, p(\cdot)}\left(\Omega^{\prime}\right)}^{2},\left\|u_{h}-u\right\|_{W^{1, p(\cdot)}\left(\Omega^{\prime}\right)}^{\gamma_{2}}\right\} \\
& \leq \frac{c\left(\gamma_{2}\right)}{\nu}\left\|u_{h}-u\right\|_{W^{1, p(\cdot)}\left(\Omega^{\prime}\right)}\left\|\mu_{h}-\mu\right\|_{W^{-1, p^{\prime}(\cdot)}\left(\Omega^{\prime}\right)} .
\end{aligned}
$$

Now, we divide by $\left\|u_{h}-u\right\|_{W^{1, p(\cdot)}\left(\Omega^{\prime}\right)}$ and since $\mu_{h} \rightarrow \mu$ in $W^{-1, p^{\prime}(\cdot)}\left(\Omega^{\prime}\right)$ we observe that indeed (4.6) holds. From here on we can proceed as in the proof of Theorem 1.4. Finally, we note that the approximation procedure is not necessary at all when $\mu \in$ $L^{1}(\Omega)$, since then the proof of Theorem 1.1 works directly - again taking into account that the convergence just takes place in the Lebesgue points. This completes the proof of Theorem 1.3.

\section{Applications and related estimates}

In this chapter we shall provide some applications of the gradient potential estimate (1.10).

5.1. $\boldsymbol{L}^{n}$-decay. We start with an application that has already been stated in Theorem 1.5 for the case that $\mu \in L^{n}$. Then, the pointwise gradient bound can be directly related to the decay properties of $r \mapsto\|\mu\|_{L^{n}\left(B_{r}\left(x_{0}\right)\right)}$ instead of the Wolff potential. Here, we give the

Proof of Theorem 1.5. By Hölder's inequality we can estimate

$$
\begin{aligned}
\left(\frac{|\mu|\left(B_{\varrho}\left(x_{0}\right)\right)}{\varrho^{n-1}}\right)^{\frac{1}{p\left(x_{0}\right)-1}} & \leq \alpha_{n}^{\frac{1}{p\left(x_{0}\right)-1}} \varrho^{\frac{1}{p\left(x_{0}\right)-1}}\left(f_{B_{\varrho}\left(x_{0}\right)}|\mu|^{n} d x\right)^{\frac{1}{n\left(p\left(x_{0}\right)-1\right)}} \\
& \leq \alpha_{n}^{\frac{n-1}{n\left(p\left(x_{0}\right)-1\right)}}\|\mu\|_{L^{n}\left(B_{\varrho}\left(x_{0}\right)\right)}^{\frac{1}{p\left(x_{0}\right)-1}},
\end{aligned}
$$

so that

$$
\mathbf{W}_{\frac{1}{p(\cdot)}, p(\cdot)}^{\mu}\left(x_{0}, \varrho\right) \leq c\left(n, \gamma_{2}\right) \int_{0}^{\varrho}\|\mu\|_{L^{n}\left(B_{r}\left(x_{0}\right)\right)}^{\frac{1}{p\left(x_{0}\right)-1}} \frac{d r}{r} .
$$

Combining the preceding estimate with (1.10) we infer the desired gradient bound in terms of the $L^{n}$-norm of $\mu$. 
5.2. Calderón and Zygmund type estimates. Our gradient estimate (1.10) allows us to deduce several types of local estimates starting with the Riesz potentialor more precisely a variable exponent version of the Riesz potential:

$$
\mathbf{I}_{\beta(\cdot)}(\mu)(x):=\int_{\mathbf{R}^{n}} \frac{d \mu(y)}{|x-y|^{n-\beta(y)}}, \quad \beta(\cdot): \mathbf{R}^{n} \rightarrow(0, n],
$$

The key is the following variable exponent version of the so called Havin-Maz'ja potential

$$
\mathbf{V}_{\frac{1}{p(\cdot)}, p(\cdot)}(|\mu|)(x):=\mathbf{I}_{\frac{1}{p(\cdot)}}\left[\left(\mathbf{I}_{\frac{1}{p(\cdot)}}(|\mu|)\right)^{\frac{1}{p(\cdot)-1}}\right](x),
$$

which is the usual potential taken pointwise - as for the Wolff potential. Then, the Wolff potential can be pointwise bounded in terms of the Havin-Maz'ja potential; see [5, Theorem 3.1], or [33]. Since the bound is pointwise it directly applies to our non standard potentials and reads as:

$$
\mathbf{W}_{\frac{1}{p(\cdot)}, p(\cdot)}^{\mu}(|\mu|)\left(x_{0}\right) \leq c\left(n, \gamma_{2}\right) \mathbf{V}_{\frac{1}{p(\cdot)}, p(\cdot)}(|\mu|)\left(x_{0}\right) .
$$

For the constant we note that due to the continuous dependence on $p$-in the constant exponent version - we can choose it in such a way that it only depends on the lower, and upper bound of $p(\cdot)$, hence on $\gamma_{2}$ (recall that $p(\cdot) \geq 2$ ), rather than on $p(\cdot)$. Therefore, we can deduce from (1.10) gradient estimates in all function spaces where the non standard Riesz potential is a bounded operator. We shall provide two particular applications here. By slight modifications of [15, Theorem 3.8] or [48] we infer that the Riesz potential $\mathbf{I}_{\beta(\cdot)}: L^{q(\cdot)}\left(\mathbf{R}^{n}\right) \rightarrow L^{\frac{n q(\cdot)}{n-\beta(\cdot) q(\cdot)}}\left(\mathbf{R}^{n}\right)$ is a bounded operator. More precisely, supposed that $q: \mathbf{R}^{n} \rightarrow\left(\gamma_{1}, \gamma_{2}\right) \subseteq(1, \infty), \beta: \mathbf{R}^{n} \rightarrow\left(\beta_{0}, \beta_{1}\right) \subseteq(0, n)$ have a modulus of continuity satisfying (2.5) and are constant outside some ball $B_{R}$ and $\|\mu\|_{L^{q(\cdot)\left(\mathbf{R}^{n}\right)}} \leq 1$, then we have

$$
\left\|\mathbf{I}_{\beta(\cdot)}(\mu)\right\|_{L^{\frac{n q(\cdot)}{n-\beta(\cdot) q(\cdot)}\left(\mathbf{R}^{n}\right)}} \leq c(q, \beta, R)\|\mu\|_{L^{q(\cdot)}\left(\mathbf{R}^{n}\right)}, \quad \text { provided that } q(x) \beta(x) \leq \tilde{n}<n .
$$

Note that $\left[15\right.$, Theorem 3.8] is for Riesz potentials $I_{\beta}$, with $\beta \equiv$ const. Nevertheless, since the proof is based on a pointwise estimate for the Riesz potential - see [15, Theorem 3.7]—we can use this estimate also for variable exponent Riesz potentials and then proceed similarly to the proof of [15, Theorem 3.8]. From the preceding estimate - which is applicable in our situation since we are working on a bounded domain $\Omega$; then the constant depends on $\operatorname{diam} \Omega$ instead of $R$-and (5.2) we infer that

$$
\mu \in L^{q(\cdot)} \Longrightarrow \mathbf{W}_{\frac{1}{p(\cdot)}, p(\cdot)}^{\mu}(\cdot, \varrho) \in L^{\frac{n q(\cdot)(p(\cdot)-1)}{n-q(\cdot)}}, \quad 1<q_{1} \leq q \leq q_{2}<n .
$$

Using this in combination with our gradient estimate (1.10) we deduce the following property of solutions as considered in Theorems 1.3 and 1.4:

$$
\mu \in L^{q(\cdot)} \Longrightarrow D u \in L_{\mathrm{loc}}^{\frac{n q(\cdot)(p(\cdot)-1)}{n-q(\cdot)}}, \quad 1<q_{1} \leq q \leq q_{2}<n,
$$

together with the related estimate. This allows us to recover the Calderón and Zygmund theory from [4] by a completely different approach and are moreover able to treat non divergence form right hand sides.

5.3. Zero order estimate. A similar technique as we used for our gradient estimate (1.10) provides an alternative proof of the zero order estimate (1.12) from [41] and updates it for general signed measures. Here, we can slightly weaken the 
hypothesis on the coefficients $a: \Omega \times \mathbf{R}^{n} \rightarrow \mathbf{R}^{n}$, assuming now instead of (1.3) and (1.4) that

$$
\left\{\begin{array}{l}
|a(x, z)| \leq L\left(|z|^{2}+s^{2}\right)^{\frac{p(x)-1}{2}}, \\
\nu\left|z_{1}-z_{2}\right|^{p(x)} \leq\left\langle a\left(x, z_{2}\right)-a\left(x, z_{1}\right), z_{2}-z_{1}\right\rangle,
\end{array}\right.
$$

for any $x \in \Omega$ and $z, z_{1}, z_{2} \in \mathbf{R}^{n}$. Moreover, we still assume (1.4), but for the modulus of continuity $\omega$ of the exponent function $p(\cdot)$ it now will be enough to suppose that the weaker condition (2.4) holds, rather than (1.6). Under these assumptions we come up with the following pointwise estimate:

$$
\left|u\left(x_{0}\right)\right| \leq c f_{B_{\varrho}\left(x_{0}\right)}|u| d x+c \mathbf{W}_{1, p(\cdot)}^{\mu}\left(x_{0}, 2 \varrho\right)+c \varrho^{2},
$$

where $c \equiv c\left(n, \nu, L, \gamma_{2},|\mu|(\Omega)\right)$.

For the sake of brevity we shall only sketch the proof. The main difference with respect to the proof of Theorem 1.1 is that now we can directly compare to solutions of the homogeneous non frozen problem (3.1), since we have a suitable zero order decay-estimate at hand. More precisely, we choose $B_{2 R} \equiv B_{2 R}\left(x_{0}\right) \subseteq \Omega$ and let $v \in W^{1, p(\cdot)}\left(B_{2 R}\right)$ be the unique weak solution to (3.1) under the assumptions (1.3), (1.4), (1.5) and (2.4). Then, there exists $\beta \in(0,1]$ and $c \geq 1$, both depending on $n, \nu, L, \gamma_{2}$, such that the estimate

$$
f_{B_{\varrho}}\left|v-(v)_{B_{\varrho}}\right| d x \leq c\left(\frac{\varrho}{R}\right)^{\beta} f_{B_{R}}\left|v-(v)_{B_{R}}\right| d x+c R
$$

holds whenever $B_{\varrho} \subseteq B_{2 R}$. The preceding estimate can be deduced from [24] and [16] as follows. First, we note that the assumptions of [24, Theorem 4.2] are a consequence of (1.3) and (1.4). Therefore, we can apply [24, Lemma 2.8] which after iterating the resulting estimate yields that

$$
\underset{B_{\varrho}}{\operatorname{Oosc}} v \leq c\left(\frac{\varrho}{R / 2}\right)^{\beta} \underset{B_{R / 2}}{\operatorname{OSc}} v+c R .
$$

Note that $\beta \in(0,1]$ and $c \geq 1$ only depend on $n, \nu, L, \gamma_{2}$. At this stage we can proceed as in the proof of [16, Proposition 4.1] and come up with (5.5).

Now, we combine (5.5) with the comparison estimate (3.4) from Lemma 3.1 to infer that

$$
\begin{aligned}
& f_{B_{\varrho}}\left|u-(u)_{B_{\varrho}}\right| d x \\
& \leq c_{1}\left(\frac{\varrho}{R}\right)^{\beta} f_{B_{2 R}}\left|v-(v)_{B_{2 R}}\right| d x+c_{1}\left(\frac{R}{\varrho}\right)^{n}\left[\left[\frac{|\mu|\left(B_{2 R}\right)}{R^{n-p_{0}}}\right]^{\frac{1}{p_{0}-1}}+R^{2}\right],
\end{aligned}
$$

with $c, c_{1} \geq 1$ depending only on $n, \nu, L, \gamma_{2},|\mu|(\Omega)$ and we have set $p_{0}=p\left(x_{0}\right)$ as usual. The preceding inequality holds whenever $B_{\varrho} \subseteq B_{2 R} \subseteq \Omega$ and is our zero order analogue of Lemma 3.6.

Next, we choose $H$ in such a way that $c_{1} / H^{\beta}=1 / 2$; then $H \geq 2$ depends only on $n, \nu, L, \gamma_{2},|\mu|(\Omega)$. Let us consider a ball $B_{R} \equiv B_{R}\left(x_{0}\right) \subseteq \Omega$. Then, we can use (5.6) with $\left(B_{\varrho}, B_{2 R}\right)$ replaced by $\left(B_{R / H}, B_{R}\right)$ to arrive at the following zero order analogue 
of (3.31):

$$
f_{B_{R / H}}\left|u-(u)_{B_{R / H}}\right| d x \leq \frac{1}{2} f_{B_{R}}\left|v-(v)_{B_{R}}\right| d x+c H^{n}\left[\left[\frac{|\mu|\left(B_{R}\right)}{R^{n-p_{0}}}\right]^{\frac{1}{p_{0}-1}}+R^{2}\right] .
$$

Now, we define the balls $B_{i}, i \in \mathbf{N}_{0}$ according to (3.32) and set $k_{i}:=\left|(u)_{B_{i}}\right|$ and $A_{i}:=f_{B_{i}}\left|u-(u)_{B_{i}}\right| d x$ and observe that we can apply (5.7) with $B_{R}=B_{i}$. From this point on we can iterate as in the proof of Theorem 1.1-but taking into account that the term involving the modulus of continuity $\omega(\cdot)$ does not appear - to conclude the proof of (5.4).

\section{Gradient continuity}

The proof of the gradient continuity result from Theorem 1.6 will heavily rely on the gradient estimate from Theorem 1.3 and the decay estimate from Lemma 3.6. Let us observe that in the present setting Theorem 1.3 directly applies, since (1.13) implies via Theorem 2.1 that $\mu$ lies in $W^{-1, p(\cdot)}\left(\Omega_{0}\right)$. Concerning Lemma 3.6 which is stated for $C^{1}$-solutions we can prove the following analogue for $W^{1, p(\cdot)}$-solutions.

Lemma 6.1. Let $u \in W^{1, p(\cdot)}(\Omega)$ be as in Theorem 1.6, then the assertion of Lemma 3.6 holds for $u$, whenever $B_{2 R} \subseteq \Omega_{0}$ such that $R \leq R_{0}$.

Proof. In order to extend the result of Lemma 3.6 to the setting of Theorem 1.6 we need an approximation argument similar to the one in Section 4.2. Observe that in the case where $\mu$ is an $L^{1}$-function rather than just a measure the proof of the lemma would directly apply. Now, let $B_{2 R} \subseteq \Omega_{0}$ be a ball as in the statement of Lemma 3.6, particularly $R \leq R_{0}$. For $h \in \mathbf{N}$ we define the mollifications $\mu_{h} \equiv \mu * \phi_{h}$ according to (4.1) and local approximations $u_{h} \in W^{1, p(\cdot)}\left(B_{R_{1}}\right)$ according to (4.5) with $\Omega^{\prime}=B_{R_{1}}$ and $R_{1}<R$. Then, from (4.6) we know that $u_{h} \rightarrow u$ in $W^{1, p(\cdot)}\left(B_{R_{1}}\right)$ and hence also in $W^{1,1}\left(B_{R_{1}}\right)$. Moreover, Lemma 3.6 applies to $u_{h}$ for any $h \in \mathbf{N}$, meaning that (3.28) holds for $u_{h}$ and $R_{1}$ instead of $u$ and $R$, respectively. Moreover, since $\mu_{h} \rightarrow \mu$ weakly in the sense of measures we have

$$
\limsup _{h \rightarrow \infty}\left|\mu_{h}\right|\left(B_{2 R_{1}}\right) \leq|\mu|\left(\overline{B_{2 R_{1}}}\right) \leq|\mu|\left(B_{2 R}\right)
$$

and therefore

$$
\begin{aligned}
& f_{B_{\varrho}}\left|D u_{h}-\left(D u_{h}\right)_{B_{\varrho}}\right| d x \\
& \leq c_{1}\left[\left(\frac{\varrho}{R_{1}}\right)^{\beta}+\left(\frac{R}{\varrho}\right)^{n}\left[L_{1} \omega\left(R_{1}\right) \log \frac{1}{R_{1}}\right]^{\frac{2}{\gamma_{2}}}\right] f_{B_{2_{1}}}\left|D u_{h}-\left(D u_{h}\right)_{B_{2 R_{1}}}\right| d x \\
& \quad+c_{1}\left(\frac{R}{\varrho}\right)^{n}\left[\left[\frac{|\mu|\left(B_{2 R}\right)}{R_{1}^{n-1}}\right]^{\frac{1}{p_{0}-1}}+\left[L_{1} \omega\left(R_{1}\right) \log \frac{1}{R_{1}}\right]^{\frac{2}{\gamma_{2}}}\left(\left|\left(D u_{h}\right)_{B_{2 R_{1}}}\right|+s\right)+R\right],
\end{aligned}
$$

with constants $\beta \in(0,1)$ and $c_{1}$ as in Lemma 3.6, independent of $h$. At this point we deduce (3.28) for $u$ by first letting $h \rightarrow \infty$-which is allowed since $u_{h} \rightarrow u$ in $W^{1,1}\left(B_{R_{1}}\right)$-and then $R_{1} \uparrow R$. This finishes the proof of the lemma.

We now come to the

Proof of Theorem 1.6. We select an arbitrary subdomain $\Omega^{\prime} \Subset \Omega_{0}$ and shall prove that $D u$ is continuous in $\Omega^{\prime}$. Furthermore, we select an intermediate domain 
$\Omega^{\prime \prime}$ such that $\Omega^{\prime} \Subset \Omega^{\prime \prime} \Subset \Omega_{0}$ and a positive radius

$$
\tilde{R} \leq \min \left\{R_{1}, \operatorname{dist}\left(\Omega^{\prime \prime}, \frac{1}{2} \partial \Omega_{0}\right), \frac{1}{2} \operatorname{dist}\left(\Omega^{\prime}, \partial \Omega^{\prime \prime}\right)\right\},
$$

where $R_{0} \equiv R_{0}\left(n, L / \nu, L_{1}, \gamma_{2}, \omega(\cdot)\right)$ denotes the radius from Lemma 6.1 , which is in turn the one from Lemma 3.6. Then, the previous choice implies whenever a ball $B_{2 R}\left(x_{0}\right)$, with $R \leq \tilde{R}$ has center $x_{0}$ in $\Omega^{\prime \prime}$, it is contained in $\Omega_{0}$, whereas when $x_{0} \in \Omega^{\prime}$ then the ball is contained in $\Omega^{\prime \prime}$. Moreover, let us observe that our hypothesis (1.13) implies

$$
\lim _{r \downarrow 0} H(R)=0, \quad \text { where } H(R):=\sup _{x \in \Omega^{\prime \prime}} \mathbf{W}_{\frac{1}{p(\cdot)}, p(\cdot)}^{\mu}(x, R) .
$$

Step 1: Du is locally bounded. This is an immediate consequence of the gradient estimates proved in the first part of the paper. More precisely, from Theorem 1.4, we know that (1.10) holds for a.e. $x_{0} \in \Omega$ and hence by a covering argument we find that

$$
|D u(x)| \leq \Upsilon \text { for a.e. } x \in \Omega^{\prime \prime},
$$

where $\Upsilon$ is a constant depending on $n, \nu, L, \gamma_{2}, M,|\mu|(\Omega)$ and $\operatorname{dist}\left(\Omega^{\prime \prime}, \partial \Omega_{0}\right)$.

Step 2: Du has vanishing mean oscillation. Here, we show that $D u \in \operatorname{VMO}\left(\Omega^{\prime}\right)$, meaning that

$$
\lim _{R \downarrow 0} \Lambda(R)=0, \quad \text { where } \Lambda(R):=\sup _{x_{0} \in \Omega^{\prime}, r \leq R} f_{B_{r}\left(x_{0}\right)}\left|D u-(D u)_{B_{r}\left(x_{0}\right)}\right| d x .
$$

For this aim let us consider an arbitrary point $x_{0} \in \Omega^{\prime}$ and $0<R \leq \tilde{R}$. Then, by our choice of $\tilde{R}$ in (6.1) we have $B_{2 R}\left(x_{0}\right) \subseteq \Omega^{\prime \prime}$ and moreover we can apply Lemma 6.1 on $B_{R}\left(x_{0}\right)$ and also on any ball contained in $B_{R}\left(x_{0}\right)$. In the following all considered balls shall be centered in $x_{0}$ and therefore we may omit the center in the notation, writing $B_{R}=B_{R}\left(x_{0}\right)$. We start as in Step 1 of the proof of Theorem 1.1, i.e., we choose $H \equiv H\left(n, \nu, L, \gamma_{2}, M,|\mu|(\Omega)\right) \geq 2$ as in (3.29) and further restrict $\tilde{R}$ in such a way that additionally to (6.1) also (3.30) with $\tilde{R}$ instead of $R_{0}$ holds. Then, applying Lemma 6.1 instead of Lemma 3.6 we infer that (3.31) also holds in our present situation. Proceeding exactly as in the proof of Theorem 1.1 and adopting the definitions (3.32), (3.33) and (3.34) from there we again arrive at (3.36). Then, taking also into account that $s \leq 1$ and $k_{i}=\left|(D u)_{B_{i}}\right| \leq \Upsilon$ for any $i \in \mathbf{N}_{0}$ by (6.3) and $\left[\omega\left(R_{i-1}\right) \log \frac{1}{R_{i-1}}\right]^{\frac{2}{p_{0}}} \leq\left[\omega\left(R_{i-1}\right) \log \frac{1}{R_{i-1}}\right]^{\frac{2}{\gamma_{2}}}$ since $\omega\left(R_{i}\right) \log \frac{1}{R_{i}} \leq 1$ by (3.30), we find that for any $i \geq 1$ there holds

$$
A_{i} \leq \frac{1}{2} A_{i-1}+c\left[\frac{|\mu|\left(B_{i-1}\right)}{R_{i-1}^{n-1}}\right]^{\frac{1}{p_{0}-1}}+c\left[\omega\left(R_{i-1}\right) \log \frac{1}{R_{i-1}}\right]^{\frac{2}{\gamma_{2}}}+c R_{i-1},
$$

where $c \equiv c\left(n, \nu, L, L_{1} \gamma_{2}, M,|\mu|(\Omega)\right)$. By induction we infer from the preceding inequality that for any $\ell \geq 1$ there holds

$$
A_{\ell} \leq\left(\frac{1}{2}\right)^{\ell} A_{0}+c \sum_{i=0}^{\ell-1}\left(\frac{1}{2}\right)^{\ell-i-1}\left[\left[\frac{|\mu|\left(B_{i}\right)}{R_{i}^{n-1}}\right]^{\frac{1}{p_{0}-1}}+\left[\omega\left(R_{i}\right) \log \frac{1}{R_{i}}\right]^{\frac{2}{\gamma_{2}}}+R_{i}\right] .
$$


Using $A_{0} \leq 2 \Upsilon$ (which follows from (6.3)), (3.38), the following variant of (3.39):

$$
\sum_{i=0}^{\ell-1}\left[\omega\left(R_{i}\right) \log \frac{1}{R_{i}}\right]^{\frac{2}{\gamma_{2}}} \leq 2 d(2 R),
$$

where $d(2 R)$ is defined in (1.6) and which can be obtained by the similar arguments as those leading to $(3.39)$ and $(3.41)$ (note that $\left(\frac{1}{2}\right)^{\ell-i-1} \leq 1$ ) we deduce from the previous inequality that

$$
A_{\ell} \leq \frac{M}{2^{\ell-2}}+c \mathbf{W}_{\frac{1}{p(\cdot)}, p(\cdot)}^{\mu}\left(x_{0}, 2 R\right)+c d(2 R)+c R .
$$

Recalling (6.2) we end up with

$$
A_{\ell}\left(x_{0}\right) \equiv A_{\ell} \leq \frac{\Upsilon}{2^{\ell-2}}+\tilde{c} H(2 R)+\tilde{c} d(2 R)+\tilde{c} R,
$$

valid for any $x_{0} \in \Omega^{\prime}$ and with a constant $\tilde{c}$ depending only on $n, \nu, L, L_{1}, \gamma_{2}, M,|\mu|(\Omega)$.

Now, let $\varepsilon>0$. We choose $\delta_{1} \leq \tilde{R}$ in such a way that

$$
\tilde{c} H(2 R)+\tilde{c} d(2 R)+\tilde{c} R \leq \frac{\varepsilon}{4^{n} H^{n}} \quad \text { whenever } R \leq \delta_{1}
$$

holds. This is possible thanks to (1.6) and (6.2). Then, $\delta_{1}$ depends on $n, \nu, L, L_{1}, \gamma_{2}$, $M, \mu(\cdot), \omega(\cdot)$ and $\varepsilon$. Next, we select $k \in \mathbf{N}$, depending on $n, \nu, L, \gamma_{2}, M,|\mu|(\Omega), \varepsilon$ such that

$$
\Upsilon \frac{\varepsilon}{2^{k-2}} \leq \frac{\varepsilon}{4^{n} H^{n}}
$$

Then, taking $R \leq \delta_{1}$ and $\ell \geq k$ we have

$$
A_{\ell}\left(x_{0}\right) \leq \frac{\varepsilon}{2^{n} H^{n}} \quad \text { for all } x_{0} \in \Omega^{\prime} \text { and } \ell \geq k .
$$

Now, we fix

$$
\delta=\frac{\delta_{1}}{H^{k}}
$$

and show that

$$
\Lambda(r)<\varepsilon \quad \text { for any } 0<r<\delta .
$$

Let $0<r<\delta$, then there exists $\ell \geq k$ such that $R / H^{\ell+1} \leq r<R / H^{\ell}$ and $r \leq \delta_{1}$ and from (6.7) we infer

$$
f_{B_{r}\left(x_{0}\right)}\left|D u-(D u)_{B_{r}\left(x_{0}\right)}\right| d x \leq 2 f_{B_{r}\left(x_{0}\right)}\left|D u-(D u)_{B_{\ell}}\right| d x \leq 2 H^{n} A_{\ell}\left(x_{0}\right)<\varepsilon .
$$

Since the previous estimate is independent of the particular point $x_{0} \in \Omega^{\prime}$ we have shown (6.8) and hence the assertion (6.4).

Step 3: Du is continuous. Here, we finally proof the continuity of $D u$ on $\Omega^{\prime}$ which in turn will be a consequence of the fact that the maps $a_{i}: \Omega^{\prime} \rightarrow \mathbf{R}^{n}$, with $i \in \mathbf{N}$

$$
a_{i}(x):=f_{B_{i}(x)} D u d y, \quad \text { where } B_{i}(x):=B_{\tilde{R} / H^{i}}(x)
$$

converge uniformly in $\Omega^{\prime}$ as $i \rightarrow \infty$. Note that $\tilde{R}$ is chosen according to Step 2 , still satisfying (6.1) and (3.30). More precisely, from Lebesgue's theorem we know that

$$
a_{i}(x) \rightarrow D u(x) \quad \text { as } i \rightarrow \infty, \quad \text { for a.e. } x \in \Omega^{\prime},
$$


and moreover, the function $x \mapsto a_{i}(x)$ is clearly continuous. Therefore, if we show that the convergence in (6.9) is uniform in $\Omega^{\prime}$, we can conclude that $D u$-or more precisely its Lebesgue representative - is continuous in $\Omega^{\prime}$. The remainder of the proof will be devoted to showing that the convergence in (6.9) indeed is uniform.

For this aim we choose $m \in \mathbf{N}$ and estimate

$$
\begin{aligned}
\left|D u(x)-a_{m}(x)\right| & =\left|\sum_{i=m}^{\infty}\left(a_{i+1}(x)-a_{i}(x)\right)\right| \\
& \leq \sum_{i=m}^{\infty} f_{B_{i+1}}\left|D u-(D u)_{B_{i}}\right| d y \leq H^{n} \sum_{i=m}^{\infty} A_{i}(x) .
\end{aligned}
$$

In order to show that the sum on the right-hand side of the preceding inequality is uniformly small, we once again take (6.5) as a starting point. Summing $(6.5)_{i}$ over $i=k, \ldots, \ell$, for $1 \leq k<\ell$ we get

$$
\sum_{i=k}^{\infty} A_{i}(x) \equiv \sum_{i=k}^{\ell} A_{i} \leq \frac{1}{2} \sum_{i=k-1}^{\ell-1} A_{i}+c \sum_{i=k-1}^{\ell-1}\left[\left[\frac{|\mu|\left(B_{i}\right)}{R_{i}^{n-1}}\right]^{\frac{1}{p_{0}-1}}+\left[\omega\left(R_{i}\right) \log \frac{1}{R_{i}}\right]^{\frac{2}{\gamma_{2}}}+R_{i}\right],
$$

where $c \equiv c\left(n, \nu, L, \gamma_{2}, L_{1}, M\right)$ and which yields after reabsorbing $\frac{1}{2} \sum_{i=k-1}^{\ell-1} A_{i}$ on the left-hand side and passing to the limit $\ell \rightarrow \infty$

$$
\begin{aligned}
\sum_{i=k}^{\infty} A_{i} & \leq A_{k-1}+2 c \sum_{i=k-1}^{\infty}\left[\left[\frac{|\mu|\left(B_{i}\right)}{R_{i}^{n-1}}\right]^{\frac{1}{p_{0}-1}}+\left[\omega\left(R_{i}\right) \log \frac{1}{R_{i}}\right]^{\frac{2}{\gamma_{2}}}+R_{i}\right] \\
& =A_{k-1}+2 c \sum_{i=0}^{\infty}\left[\left[\frac{|\mu|\left(B_{i+k-1}\right)}{R_{i+k-1}^{n-1}}\right]^{\frac{1}{p_{0}-1}}+\left[\omega\left(R_{i+k-1}\right) \log \frac{1}{R_{i+k-1}}\right]^{\frac{2}{\gamma_{2}}}+R_{i+k-1}\right],
\end{aligned}
$$

where we have denoted for the moment $B_{i} \equiv B_{i}(x)$. Using (3.38), (6.6) and (3.41) for $B_{k-1}(x) \equiv B_{\tilde{R} / H^{k-1}}(x)$ instead of $B_{R}$ in the preceding inequality and then recalling the notation from (6.4) and (6.2) we find that

$$
\begin{aligned}
\sum_{i=k}^{\infty} A_{i}(x) & \leq A_{k-1}(x)+c \mathbf{W}_{\frac{1}{p(\cdot)}, p(\cdot)}^{\mu}\left(x, \frac{\tilde{R}}{H^{k-2}}\right)+c d\left(\frac{\tilde{R}}{H^{k-2}}\right)+\frac{c \tilde{R}}{H^{k-2}} \\
& \leq \Lambda\left(\frac{\tilde{R}}{H^{k-1}}\right)+c H\left(\frac{\tilde{R}}{H^{k-2}}\right)+c d\left(\frac{\tilde{R}}{H^{k-2}}\right)+\frac{c \tilde{R}}{H^{k-2}}
\end{aligned}
$$

where $c \equiv c\left(n, \nu, L, L_{1}, \gamma_{2}, M,|\mu|(\Omega)\right)$. Note that the previous inequality holds uniformly in $x \in \Omega^{\prime}$ and therefore due to (6.4), (6.2) and (1.6), for any $\varepsilon>0$ we can find $k \in \mathbf{N}$ large enough-depending on $n, \nu, L, L_{1}, \gamma_{2}, M, H,|\mu|(\Omega), \varepsilon$ and since $H \equiv H\left(n, \nu, L, \gamma_{2}, M,|\mu|(\Omega)\right)$ ultimately on $n, \nu, L, \gamma_{2}, L_{1}, M,|\mu|(\Omega), \varepsilon$ - to ensure that

$$
\sum_{i=k}^{\infty} A_{i}(x)<\frac{\varepsilon}{H^{n}} \quad \text { for any } x \in \Omega^{\prime},
$$

which in turn by (6.10) implies

$$
\left|D u(x)-a_{m}(x)\right| \leq H^{n} \sum_{i=m}^{\infty} A_{i}(x) \leq H^{n} \sum_{i=k}^{\infty} A_{i}(x)<\varepsilon, \quad \text { for every } m \geq k .
$$


This finally shows that the convergence in (6.9) indeed is uniform and therefore the desired continuity of $D u$, completing the proof of the theorem.

Proof of Theorem 1.7. From (1.15) we infer that

$$
\sup _{x \in \Omega} \mathbf{W}_{\frac{1}{p(\cdot)}, p(\cdot)}^{\mu}(x, \varrho) \leq \int_{0}^{\varrho} h(r) \frac{d r}{r} .
$$

Now, the assumption (1.14) ensures that (1.13) is satisfied and therefore the asserted gradient continuity immediately follows from Theorem 1.6.

Proof of Theorem 1.8. This follows from Theorem 1.6, since (5.1) together with (1.16) ensures us that the hypothesis (1.13) of Theorem 1.6 is in force.

\section{References}

[1] ACERBi, E., and G. Mingione: Regularity results for a class of functionals with non-standard growth. - Arch. Ration. Mech. Anal. 156, 2001, 121-140.

[2] Acerbi, E., and G. Mingione: Regularity results for a class of quasiconvex functionals with nonstandard growth. - Ann. Sc. Norm. Super. Pisa Cl. Sci. (4) 30, 2001, 311-339.

[3] Acerbi, E., and G. Mingione: Regularity results for stationary electro-rheological fluids. Arch. Ration. Mech. Anal. 164, 2002, 213-259.

[4] Acerbi, E., G. Mingione: Gradient estimates for the $p(x)$-Laplacean system. - J. Reine Angew. Math. 584, 2005, 117-148.

[5] Adams, D. R., and N. G. Meyers: Thinness and Wiener criteria for non-linear potentials. Indiana Univ. Math. J. 22, 1972, 169-197.

[6] Alkhutov, Y., and O. Krasheninnikova: Continuity at boundary points of solutions of quasilinear elliptic equations with nonstandard growth condition. - Izv. Ross. Akad. Nauk Ser. Mat. 68:6, 2004, 3-60; English transl. in Izv. Math. 68:6, 2004, 1063-1117.

[7] Astala, K., T. Iwaniec, P. Koskela, and G. Martin: Mappings of BMO-bounded distortion. - Math. Ann. 317, 2000, 703-726.

[8] Bendahmane, M., and P. Wittbold: Renormalized solutions for nonlinear elliptic equations with variable exponents and $L^{1}$ data. - Nonlinear Anal. 70:2, 2009, 567-583.

[9] Boccardo, L., and T. GallouËT: Nonlinear elliptic and parabolic equations involving measure data. - J. Funct. Anal. 87:1, 1989, 149-169.

[10] Boccardo, L., and T. Gallouët: Nonlinear elliptic equations with right-hand side measures. - Comm. Partial Differential Equations 17:3-4, 1992, 641-655.

[11] Bögelein, V., and A. Zatorska-Goldstein: Higher integrability of very weak solutions of systems of $p(x)$-Laplacean type. - J. Math. Anal. Appl. 336:1, 2007, 480-497.

[12] Chen, Y., S. Levine, and M. RaO: Variable exponent, linear growth functionals in image restoration. - SIAM J. Appl. Math. 66:4, 2006, 1383-1406.

[13] Coscia, A., and G. Mingione: Hölder continuity of the gradient of $p(x)$ harmonic mappings. - C. R. Acad. Sci. Paris 328:1, 1999, 363-368.

[14] Dall'Aglio, A.: Approximated solutions of equations with $L^{1}$ data. Application to the $H$ convergence of quasi-linear parabolic equations. - Ann. Mat. Pura Appl. (4) 170, 1996, 207-240.

[15] Diening, L.: Riesz potential and Sobolev embeddings on generalized Lebesgue and Sobolev spaces $L^{p(\cdot)}$ and $W^{k, p(\cdot)}$. - Math. Nachr. 268, 2004, 31-43.

[16] Duzanr, F., and G. Mingione: Gradient estimates via non-linear potentials. - Amer. J. Math. (to appear). 
[17] Duzane, F., and G. Mingione: Gradient continuity estimates. - Calc. Var. Partial Differential Equations (to appear), doi:10.1007/s00526-010-0314-6.

[18] Duzane, F., and G. Mingione: Lipschitz estimates for degenerate elliptic systems. - To appear.

[19] Edmunds, D., and J. RÁKosník: Sobolev embeddings with variable exponent. - Stud. Math. 143:3, 2000, 267-293.

[20] Edmunds, D., and J. RÁkosník: Sobolev embeddings with variable exponent II. - Math. Nachr. 246/247, 2002, 53-67.

[21] Eleuteri, M.: Hölder continuity results for a class of functionals with non standard growth. - Boll. Unione Mat. Ital. Sez. B 8:7, 2004, 129-157.

[22] Esposito, L., F. Leonetti, and G. Mingione: Sharp regularity for functionals with $(p, q)$ growth. - J. Differential Equations 204:1, 2004, 5-55.

[23] FAn, X.: Global $C^{1, \alpha}$ regularity for variable exponent elliptic equations in divergence form. J. Differential Equations 235:2, 2007, 397-417.

[24] FAn, X., and D. Zhaо: A class of De Giorgi type and Hölder continuity. - Nonlinear Anal. 36:3(A), 1999, 295-318.

[25] Giusti, E.: Direct methods in the calculus of variations. - World Scientific, Singapore, 2003.

[26] Habermann, J.: Partial regularity for minima of higher order functionals with $p(x)$ growth. - Manuscripta Math. 126:1, 2008, 1-40.

[27] Habermann, J.: Calderón-Zygmund estimates for higher order systems with $p(x)$ growth. Math. Z. 258:2, 2008, 427-462.

[28] Habermann, J., and A. Zatorska-Goldstein: Regularity for minimizers of functionals with nonstandard growth by $A$-harmonic approximation. - Nonlinear Differential Equations Appl. 15, 2008, 169-194.

[29] Harjulehto, P., P. Hästö, Ú. Văn Lê, and M. Nuortio: Overview of differential equations with non-standard growth. - Nonlinear Anal. 72:12, 2010, 4551-4574.

[30] HÄsт̈̈, P.: Counter examples of regularity in variable exponent Sobolev spaces. - Contemp. Math. 370, 2005, 133-143.

[31] Hästö, P.: On the density of smooth functions in variable exponent Sobolev space. - Rev. Mat. Iberoamericana 23:1, 2007, 215-237.

[32] HästÖ, P.: The maximal function on Lebesgue spaces with variable exponent approaching 1. - Math. Nachr. 280:1-2, 2007, 74-82.

[33] Havin, M., and V. G. MAZ'JA: A nonlinear potential theory. - Russ. Math. Surveys 27, 1972, $7-64$.

[34] HudzIK, H.: The problems of separability, duality, reflexivity and of comparison for generalized Orlicz-Sobolev spaces $W_{M}^{k}(\Omega)$. - Comment. Math. Prace Mat. 21, 1979, 315-324.

[35] Iwaniec, T.: p-harmonic tensors and quasiregular mappings. - Ann. of Math. (2) 136:3, 1992, 589-624.

[36] Iwaniec, T., and A. Verde: On the operator $\mathscr{L}(f)=f \log |f|$. - J. Funct. Anal. 169, 1999, $391-420$.

[37] KilpeläInen, T., and J. MalÝ: Degenerate elliptic equations with measure data and nonlinear potentials. - Ann. Sc. Norm. Super. Pisa Cl. Sci. (4) 19:4, 1992, 591-613.

[38] Kilpeläinen, T., and J. MalÝ: The Wiener test and potential estimates for quasilinear elliptic equations. - Acta Math. 172, 1994, 137-161.

[39] KováC̆IK, O., and J. RÁkosník: On spaces $L^{p(x)}$ and $W^{k, p(x)}$ - Czechoslovak Math. J. 41:116, 1991, 592-618. 
[40] LUKKARI, T.: Elliptic equations with nonstandard growth involving measures. - Hiroshima Math. J. 38(1) (2008), 155-176.

[41] Lukkari, T., F. Maeda, and N. Marola: Wolff potential estimates for elliptic equations with nonstandard grwoth and applications. - Forum Math. (to appear), doi:10.1515/FORUM. 2010.057 .

[42] Marcellini, P.: Regularity and existence of solutions of elliptic equations with $p, q$-growth conditions. - J. Differential Equations 90, 1991, 1-30.

[43] Mingione, G.: The Calderón-Zygmund theory for elliptic problems with measure data. - Ann. Sc. Norm. Super. Pisa Cl. Sci. (5) 6, 2007, 195-261.

[44] Mingione, G.: Gradient estimates below the duality exponent. - Math. Ann. 346, 2010, 571627.

[45] Mingione, G.: Gradient potential estimates. - J. Eur. Math. Soc. (to appear).

[46] Prignet, A.: Remarks on existence and uniqueness of solutions of elliptic problems with right-hand side measures. - Rend. Mat. Appl. (7) 15:3, 1995, 321-337.

[47] Rajagopal, K. R., and M. RŮŽIČKA: Mathematical modelling of electro-rheological fluids. Contin. Mech. Thermodyn. 13, 2001, 59-78.

[48] Samko, S. G.: Convolution and potential type operators in $L^{p(x)}\left(\mathbf{R}^{n}\right)$. - Integral Transforms Spec. Funct. 7:3-4, 1998, 261-284.

[49] Sanchón, M., and J. M. Urbano: Entropy solutions for the $p(x)$-Laplace equation. - Trans. Amer. Math. Soc. 361, 2009, 6387-6405.

[50] Serrin, J.: Pathological solutions of elliptic differential equations. - Ann. Sc. Norm. Super. Pisa Sci. Fis. Mat. (3) 18, 1964, 385-387.

[51] Trudinger, N. S., and X. J. WAng: On the weak continuity of elliptic operators and applications to potential theory. - Amer. J. Math. 124, 2002, 369-410.

[52] Zhikov, V. V.: On some variational problems. - Russian J. Math. Phys. 5, 1997, 105-116.

[53] Zhikov, V. V., and S. E. PAstukhova: Improved integrability of the gradients of solutions of elliptic equations with variable nonlinearity exponent. - Sb. Math. 199:12, 2008, 1751-1782. 